\title{
The non-uniqueness of solution for initial value problem of impulsive differential equations involving higher order Katugampola fractional derivative
}

\section{Xian-Min Zhang ${ }^{1 *}$ (D)}

"Correspondence:

XianminZhang@126.com; z6x2m@126.com

'School of Mathematics and Statistics, Yangtze Normal

University, Chongqing, P.R. China

\section{Springer}

\begin{abstract}
In this paper we consider the initial value problem for some impulsive differential equations with higher order Katugampola fractional derivative (fractional order $q \in(1,2])$. The systems of impulsive higher order fractional differential equations can involve one or two kinds of impulses, and by analyzing the error between the approximate solution and exact solution it is found that these impulsive systems are equivalent to some integral equations with one or two undetermined constants correspondingly, which uncover the non-uniqueness of solution to these impulsive systems. Some numerical examples are offered to explain the obtained results.
\end{abstract}

MSC: $34 \mathrm{~A} 08 ; 34 \mathrm{~A} 37$

Keywords: Fractional differential equations; Impulsive fractional differential equations; Generalized fractional derivative; Non-uniqueness of solution

\section{Introduction}

Fractional calculus serves as an important tool to characterize hereditary properties in many fields of science and engineering (such as chaotic behavior, epidemiology, thermal science, hydrology, and biology [1-17]). Since fractional calculus was put forward in the seventeenth century, there have appeared several definitions of fractional derivatives: Riemann-Liouville, Caputo, Hadamard, Grunwald-Letnikov etc. [18, 19]. To unify these fractional derivatives, some generalized fractional operators (such as Erdélyi-Kober fractional operator [18], Hilfer fractional operator [20, 21], Katugampola fractional operator [22, 23], and Atangana-Baleanu fractional operator [24] etc.) were presented, and some properties of these generalized fractional operators and differential equations involving these generalized fractional derivatives were widely studied [25-33]. The potential application in quantum mechanics was considered for some properties of the Katugampola fractional derivative in [34], and the existence and uniqueness of solutions was studied for fractional Langevin equation with the nonlocal Katugampola fractional integral conditions in [35].

(c) The Author(s) 2020. This article is licensed under a Creative Commons Attribution 4.0 International License, which permits use, sharing, adaptation, distribution and reproduction in any medium or format, as long as you give appropriate credit to the original author(s) and the source, provide a link to the Creative Commons licence, and indicate if changes were made. The images or other third party material in this article are included in the article's Creative Commons licence, unless indicated otherwise in a credit line to the material. If material is not included in the article's Creative Commons licence and your intended use is not permitted by statutory regulation or exceeds the permitted use, you will need to obtain permission directly from the copyright holder. To view a copy of this licence, visit http://creativecommons.org/licenses/by/4.0/. 
Furthermore, impulsive differential equations are used in description of some processes with impulsive effects [36], and the subject of impulsive fractional differential equations (IFrDE) has been getting an enormous amount of attention recently [37-45]. In addition, IFrDE was considered from the short memory case that addressed the nonlocality and the impulsive conditions in [46]. For the studies of IFrDE, most of them considered impulsive differential equations involving the Caputo type fractional derivative, and a few of them were concerned with impulsive non-Caputo type fractional differential equations. Therefore, we consider the equivalent integral equation for the initial value problem (IVP) of impulsive differential equations involving higher order non-Caputo type fractional derivative (in the sense of Katugampola):

$$
\left\{\begin{array}{l}
{ }_{t_{0}}^{K} \mathcal{D}_{t}^{q, \rho} x(t)=f(t, x(t)), \\
\quad t \in\left(t_{0}, T\right], t \neq t_{k}(k=1,2, \ldots, m) \text { and } t \neq \bar{t}_{l}(l=1,2, \ldots, n), \\
{ }_{t_{0}} \mathcal{D}_{t_{k}+\rho}^{q-1, \rho} x\left(t_{k}+\right)-{ }_{t_{0}}^{K} \mathcal{D}_{t_{k}-}^{q-1, \rho} x\left(t_{k}-\right)=J_{k}\left(x\left(t_{k}-\right)\right), \quad k=1,2, \ldots, m, \\
{ }_{t_{0}}^{K} \mathcal{I}_{\bar{t}_{l^{+}}-{ }^{2}}^{2-\rho} x\left(\bar{t}_{l^{+}}\right)-{ }_{{ }_{0}}^{K} \mathcal{I}_{\bar{t}_{l^{-}}}^{2-q, \rho} x\left(\bar{t}_{l^{-}}\right)=\bar{J}_{l}\left(x\left(\bar{t}_{l^{-}}\right)\right), \quad l=1,2, \ldots, n, \\
\left.{ }_{{ }_{t_{0}}} \mathcal{D}_{t}^{q-1, \rho} x(t)\right|_{t \rightarrow t_{0^{+}}}=x_{1},\left.\quad{ }_{t_{0}}^{K} \mathcal{I}_{t}^{2-q, \rho} x(t)\right|_{t \rightarrow t_{0^{+}}} ^{2-}=x_{2},
\end{array}\right.
$$

where ${ }_{t_{0}}^{K} \mathcal{D}_{t}^{q, \rho}$ (here $q \in(1,2]$ and $\rho>0$ ) denotes the left-sided Katugampola fractional derivative of order $q .{ }_{t_{0}}^{K} \mathcal{D}_{t_{k}+}^{q-1, \rho} x\left(t_{k}+\right)=\lim _{\varepsilon \rightarrow 0+}{ }_{t_{0}}^{K} \mathcal{D}_{t_{k}+\varepsilon}^{q-1, \rho} x\left(t_{k}+\varepsilon\right)$ and ${ }_{t_{0}}^{K} \mathcal{D}_{t_{k^{-}}}^{q-1, \rho} x\left(t_{k^{-}}\right)=$ $\lim _{\varepsilon \rightarrow 0-}{ }_{t_{0}}^{K} \mathcal{D}_{t_{k}+\varepsilon}^{q-1, \rho} x\left(t_{k}+\varepsilon\right)$ represent the right and left limits of ${ }_{t_{0}}^{K} \mathcal{D}_{t}^{q-1, \rho} x(t)$ at $t=t_{k}$, respectively. ${ }_{t_{0}}^{K} \mathcal{I}_{\bar{t}_{l}+}^{2-q, \rho} x\left(\bar{t}_{l}+\right)$ and ${ }_{t_{0}}^{K} \mathcal{I}_{\bar{t}_{l}-}^{2-q, \rho} x\left(\bar{t}_{l}-\right)$ denote the right and left limits of ${ }_{t_{0}}^{K} \mathcal{I}_{t}^{2-q, \rho} x(t)$ at $t=\bar{t}_{l}$, respectively. Two kinds of impulsive points satisfy $0 \leq t_{0}<t_{1}<\cdots<t_{m}<t_{m+1}=T$ and $t_{0}<\bar{t}_{1}<\cdots<\bar{t}_{n}<\bar{t}_{n+1}=T$, respectively. Moreover, for these impulsive points, two assumptions are given as follows:

(H1) Let $\left\{t_{0}, t_{1}, t_{2}, \ldots, t_{m}, \bar{t}_{1}, \bar{t}_{2}, \ldots, \bar{t}_{n}, T\right\}=\left\{t_{0}, t_{1}^{\prime}, t_{2}^{\prime}, \ldots, t_{M}^{\prime}, T\right\}$ satisfy

$$
0 \leq t_{0}<t_{1}^{\prime}<t_{2}^{\prime}<\cdots<t_{M}^{\prime}<t_{M+1}^{\prime}=T \text {. }
$$

(H2) For each $\left[t_{0}, t_{k}^{\prime}\right](k=1,2, \ldots, M)$, suppose $\left[t_{0}, t_{k_{1}}\right] \subseteq\left[t_{0}, t_{k}^{\prime}\right] \subset\left[t_{0}, t_{k_{1}+1}\right]$ (here $\left.k_{1} \in\{1,2, \ldots, m\}\right)$ and $\left[t_{0}, \bar{t}_{k_{2}}\right] \subseteq\left[t_{0}, t_{k}^{\prime}\right] \subset\left[t_{0}, \bar{t}_{k_{2}+1}\right]$ (here $k_{2} \in\{1,2, \ldots, n\}$ ), respectively.

In particular, letting $J_{k}\left(x\left(t_{k}-\right)\right)=0$ (for all $\left.k \in\{1,2, \ldots, m\}\right)$ and $\bar{J}_{l}\left(x\left(\bar{t}_{l}-\right)\right)=0$ (for all $l \in$ $\{1,2, \ldots, n\})$ in $(1.1)$ respectively, we obtain two simple impulsive systems:

$$
\left\{\begin{array}{l}
{ }_{t_{0}}^{K} \mathcal{D}_{t}^{q, \rho} x(t)=f(t, x(t)), \quad t \in\left(t_{0}, T\right] \text { and } t \neq t_{k}(k=1,2, \ldots, m), \\
{ }_{t_{0}} \mathcal{D}_{t_{k^{+}}}^{q-1, \rho} x\left(t_{k}+\right)-{ }_{t_{0}}^{K} \mathcal{D}_{t_{k}-}^{q-1, \rho} x\left(t_{k}-\right)=J_{k}\left(x\left(t_{k}-\right)\right), \quad k=1,2, \ldots, m, \\
\left.{ }_{t_{0}}^{K} \mathcal{D}_{t}^{q-1, \rho} x(t)\right|_{t \rightarrow t_{0^{+}}}=x_{1},\left.\quad{ }_{t_{0}}^{K} \mathcal{I}_{t}^{2-q, \rho} x(t)\right|_{t \rightarrow t_{0^{+}}}=x_{2}
\end{array}\right.
$$

and

$$
\left\{\begin{array}{l}
{ }_{t_{0}}^{K} \mathcal{D}_{t}^{q, \rho} x(t)=f(t, x(t)), \quad t \in\left(t_{0}, T\right] \text { and } t \neq \bar{t}_{l}(l=1,2, \ldots, n), \\
{ }_{t_{0}} \mathcal{I}_{\bar{t}_{l^{+}}}^{2-q, \rho} x\left(\bar{t}_{l^{+}}\right)-{ }_{t_{0}}^{K} \mathcal{I}_{\bar{t}_{l^{-}}}^{2-q, \rho} x\left(\bar{t}_{l^{-}}\right)=\bar{J}_{l}\left(x\left(\bar{t}_{l^{-}}\right)\right), \quad l=1,2, \ldots, n, \\
\left.{ }_{t_{0}}^{K} \mathcal{D}_{t}^{q-1, \rho} x(t)\right|_{t \rightarrow t_{0^{+}}}=x_{1},\left.\quad{ }_{t_{0}}^{K} \mathcal{I}_{t}^{2-q, \rho} x(t)\right|_{t \rightarrow t_{0^{+}}}=x_{2} .
\end{array}\right.
$$


Moreover, letting $\left\{t_{1}, t_{2}, \ldots, t_{m}\right\}=\left\{\bar{t}_{1}, \bar{t}_{2}, \ldots, \bar{t}_{n}\right\}$ in (1.1), we get the impulsive system

$$
\left\{\begin{array}{l}
{ }_{t_{0}}^{K} \mathcal{D}_{t}^{q, \rho} x(t)=f(t, x(t)), \quad t \in\left(t_{0}, T\right] \text { and } t \neq t_{k}(k=1,2, \ldots, m), \\
{ }_{t_{0}} \mathcal{D}_{t_{k}+}^{q-1, \rho} x\left(t_{k}+\right)-{ }_{t_{0}}^{K} \mathcal{D}_{t_{k}-}^{q-1, \rho} x\left(t_{k}-\right)=J_{k}\left(x\left(t_{k}-\right)\right), \quad k=1,2, \ldots, m, \\
{ }_{t_{0}} \mathcal{I}_{t_{k^{+}}}^{2-q, \rho} x\left(t_{k}+\right)-{ }_{t_{0}}^{K} \mathcal{I}_{t_{k^{-}}}^{2-q, \rho} x\left(t_{k}-\right)=\bar{J}_{k}\left(x\left(t_{k}-\right)\right), \quad k=1,2, \ldots, m, \\
\left.{ }_{t_{0}} \mathcal{D}_{t}^{q-1, \rho} x(t)\right|_{t \rightarrow t_{0^{+}}}=x_{1},\left.\quad{ }_{t_{0}}^{K} \mathcal{I}_{t}^{2-q, \rho} x(t)\right|_{t \rightarrow t_{0^{+}}}=x_{2} .
\end{array}\right.
$$

Next we introduce some basic definitions and conclusions regarding the Katugampola fractional derivative in Sect. 2 and give some properties of IFrDEs (1.1)-(1.3) in Sect. 3. Then, we seek the equivalent integral equations of IFrDEs (1.1)-(1.4) in Sect. 4. Finally, we use some numerical examples to expound the obtained results in Sect. 5.

\section{Preliminaries}

Let $[a, b](-\infty \leq a<b<\infty)$ be a finite interval on the real axis $\mathbb{R}$ and $C[a, b]$ be the set of continuous functions on $[a, b]$. Define the function space

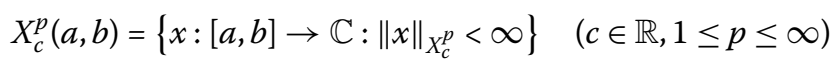

endowed with the norm $\|x\|_{X_{c}^{p}}=\left(\int_{a}^{b}\left|t^{c} x(t)\right|^{p} \frac{d t}{t}\right)^{1 / p} \quad(1 \leq p<\infty)$ and $\|x\|_{X_{c}^{\infty}}=$ ess $\sup _{t \in[a, b]}\left[t^{c}|x(t)|\right]$.

Definition 2.1 ([22]) The left-sided Katugampola fractional integrals of order $\alpha \in \mathbb{C}$ $(\Re(\alpha)>0)$ of function $x \in X_{c}^{p}(a, b)$ are defined by

$$
\left({ }_{a}^{K} \mathcal{I}_{t}^{\alpha, \rho} x\right)(t)=\frac{1}{\Gamma(\alpha)} \int_{a}^{t}\left(\frac{t^{\rho}-s^{\rho}}{\rho}\right)^{\alpha-1} \frac{x(s) d s}{s^{1-\rho}} \quad(t>a \geq 0)
$$

Definition 2.2 ([23]) The left-sided Katugampola fractional derivatives of order $\alpha \in \mathbb{C}$ $(\Re(\alpha)>0)$ are defined by

$$
\begin{aligned}
\left({ }_{a}^{K} \mathcal{D}_{t}^{\alpha, \rho} x\right)(t) & =\gamma^{n}\left({ }_{a}^{K} \mathcal{I}_{t}^{n-\alpha, \rho} x\right)(t) \\
& =\frac{\gamma^{n}}{\Gamma(n-\alpha)} \int_{a}^{t}\left(\frac{t^{\rho}-s^{\rho}}{\rho}\right)^{n-\alpha-1} \frac{x(s) d s}{s^{1-\rho}} \\
(\rho>0, t & \left.>a \geq 0, \gamma=t^{1-\rho} \frac{d}{d t}\right) .
\end{aligned}
$$

Remark 2.3 From the L'Hospital rule, we have $\lim _{\rho \rightarrow 0+}\left(\frac{t^{\rho}-\tau^{\rho}}{\rho}\right)^{q-1}=\left(\ln \frac{t}{\tau}\right)^{q-1}$. The Katugampola fractional operators with $\rho \rightarrow 0+$ and $\rho=1$ are the Hadamard fractional operator and the Riemann-Liouville fractional operator, respectively.

For $n-1<\alpha \leq n(n \in \mathbb{N})$, a weighted space of continuous functions is defined by

$$
\begin{aligned}
& C_{n-\alpha, \rho}[a, b]=\left\{x(t):\left(t^{\rho}-a^{\rho}\right)^{n-\alpha} x(t) \in C[a, b],\|x\|_{C_{n-\alpha, \rho}}=\left\|\left(t^{\rho}-a^{\rho}\right)^{n-\alpha} x(t)\right\|_{C}\right\} \\
& \quad(\rho \neq 0)
\end{aligned}
$$


and

$$
\begin{array}{r}
C_{n-\alpha, 0}[a, b]=\left\{x(t):(\ln t-\ln a)^{n-\alpha} x(t) \in C[a, b],\right. \\
\left.\|x\|_{C_{n-\alpha, 0}}=\left\|(\ln t-\ln a)^{n-\alpha} x(t)\right\|_{C}\right\} .
\end{array}
$$

Moreover, let

$$
C_{n-\alpha, \rho}^{\alpha}[a, b]=\left\{x(t) \in C_{n-\alpha, \rho}[a, b]:{ }_{a}^{K} \mathcal{D}_{t}^{\alpha, \rho} x(t) \in C_{n-\alpha, \rho}[a, b]\right\}
$$

and

$$
C_{2-\alpha, \rho}^{2}[a, T]=\left\{x(t) \in C[a, b]: \gamma^{2} x(t) \in C_{2-\alpha, \rho}[a, b], \gamma=t^{1-\rho} \frac{d}{d t}\right\}
$$

Lemma 2.4 Let $q \in(1,2]$ and $a, \rho>0$, and let $f:[a, T] \times \mathbb{R} \rightarrow \mathbb{R}$ be a function such that $f(\cdot, x(\cdot)) \in C_{2-q, \rho}[a, T]$ for any $x(\cdot) \in C_{2-q, \rho}[a, T]$.

If $x(\cdot) \in C_{2-q, \rho}^{q}[a, T]$, then $x(t)$ is a solution of the fractional differential equation

$$
\left\{\begin{array}{l}
{ }_{a}^{K} \mathcal{D}_{t}^{q, \rho} x(t)=f(t, x(t)), \quad t \in(a, T], \\
\left.{ }_{a}^{K} \mathcal{D}_{t}^{q-1, \rho} x(t)\right|_{t \rightarrow a+}=x_{1},\left.\quad{ }_{a}^{K} \mathcal{I}_{t}^{2-q, \rho} x(t)\right|_{t \rightarrow a+}=x_{2}
\end{array}\right.
$$

if, and only if, $x(t)$ satisfies the integral equation

$$
\begin{aligned}
& x(t)= \frac{x_{2}}{\Gamma(q-1)}\left[\frac{t^{\rho}-a^{\rho}}{\rho}\right]^{q-2}+\frac{x_{1}}{\Gamma(q)}\left[\frac{t^{\rho}-a^{\rho}}{\rho}\right]^{q-1} \\
&+\frac{1}{\Gamma(q)} \int_{a}^{t}\left[\frac{t^{\rho}-\tau^{\rho}}{\rho}\right]^{q-1} \frac{f d \tau}{\tau^{1-\rho}} \\
& \text { for } t \in(a, T] \text { and } f=f(\tau, x(\tau)) .
\end{aligned}
$$

Proof First, we prove the necessity. Let $x(t) \in C_{2-q, \rho}^{q}[a, T]$ be a solution of (2.8). By the hypotheses $x(t) \in C_{2-q, \rho}^{q}[a, T]$ and ${ }_{a}^{K} \mathcal{D}_{t}^{q, \rho} x(t)=\gamma^{2}\left({ }_{a}^{K} \mathcal{I}_{t}^{2-q, \rho} x\right)(t)$, we have ${ }_{a}^{K} \mathcal{I}_{t}^{2-q, \rho} x(t) \in C[a, T]$ and ${ }_{a}^{K} \mathcal{I}_{t}^{2-q, \rho} x \in C_{2-q, \rho}^{2}[a, T]$. Therefore, by (2.8), we get

$$
{ }_{a}^{K} \mathcal{D}_{t}^{q, \rho} x(t)=\left(t^{1-\rho} \frac{d}{d t}\right)^{2}\left({ }_{a}^{K} \mathcal{I}_{t}^{2-q, \rho} x(t)\right)=f(t, x(t)) .
$$

Therefore

$$
{ }_{a}^{K} \mathcal{I}_{t}^{2-q, \rho} x(t)=x_{2}+\frac{t^{\rho}-a^{\rho}}{\rho} x_{1}+\int_{a}^{t} \frac{t^{\rho}-\tau^{\rho}}{\rho} \frac{f d \tau}{\tau^{1-\rho}} .
$$

Applying the operator ${ }_{a}^{K} \mathcal{I}_{t}^{q, \rho}$ to two sides of (2.10), we have

$$
\begin{aligned}
{ }_{a}^{K} \mathcal{I}_{t}^{2, \rho} x(t)= & \frac{x_{2}}{\Gamma(q+1)}\left[\frac{t^{\rho}-a^{\rho}}{\rho}\right]^{q}+\frac{x_{1}}{\Gamma(q+2)}\left[\frac{t^{\rho}-a^{\rho}}{\rho}\right]^{q+1} \\
& +\frac{1}{\Gamma(q+2)} \int_{a}^{t}\left[\frac{t^{\rho}-\tau^{\rho}}{\rho}\right]^{q+1} \frac{f d \tau}{\tau^{1-\rho}}
\end{aligned}
$$


Using the operator $\gamma^{2}$ to two sides of (2.11), we obtain

$$
\begin{aligned}
x(t)= & \frac{x_{2}}{\Gamma(q-1)}\left[\frac{t^{\rho}-a^{\rho}}{\rho}\right]^{q-2}+\frac{x_{1}}{\Gamma(q)}\left[\frac{t^{\rho}-a^{\rho}}{\rho}\right]^{q-1} \\
& +\frac{1}{\Gamma(q)} \int_{a}^{t}\left[\frac{t^{\rho}-\tau^{\rho}}{\rho}\right]^{q-1} \frac{f d \tau}{\tau^{1-\rho}}
\end{aligned}
$$

for $t \in(a, T]$.

Now we prove the sufficiency. Let $x(t) \in C_{2-q, \rho}^{q}[a, T]$ satisfy Eq. (2.9), which can be written as (2.9). Moreover, by the hypotheses of Lemma 2.4 , for any $x(\cdot) \in C_{2-q, \rho}[a, T]$, we have $f(\cdot, x(\cdot)) \in C_{2-q, \rho}[a, T]$. Applying the operators ${ }_{a}^{K} \mathcal{D}_{t}^{q, \rho},{ }_{a}^{K} \mathcal{D}_{t}^{q-1, \rho}$, and ${ }_{a}^{K} \mathcal{I}_{t}^{2-q, \rho}$ to both sides of (2.9), respectively, we obtain

$$
\begin{aligned}
& { }_{a}^{K} \mathcal{D}_{t}^{q, \rho} x(t)={ }_{a}^{K} \mathcal{D}_{t}^{q, \rho}\left\{\frac{x_{2}}{\Gamma(q-1)}\left[\frac{t^{\rho}-a^{\rho}}{\rho}\right]^{q-2}+\frac{x_{1}}{\Gamma(q)}\left[\frac{t^{\rho}-a^{\rho}}{\rho}\right]^{q-1}\right. \\
& \left.+\frac{1}{\Gamma(q)} \int_{a}^{t}\left[\frac{t^{\rho}-\tau^{\rho}}{\rho}\right]^{q-1} \frac{f d \tau}{\tau^{1-\rho}}\right\} \\
& =f(t, x(t)) \quad \text { for } t \in(a, T] \text {, } \\
& { }_{a}^{K} \mathcal{D}_{t}^{q-1, \rho} x(t)=x_{1}+\int_{a}^{t} \frac{f d \tau}{\tau^{1-\rho}} \quad \text { for } t \in(a, T],
\end{aligned}
$$

and

$$
{ }_{a}^{K} \mathcal{I}_{t}^{2-q, \rho} x(t)=x_{2}+\frac{t^{\rho}-a^{\rho}}{\rho} x_{1}+\int_{a}^{t} \frac{t^{\rho}-\tau^{\rho}}{\rho} \frac{f d \tau}{\tau^{1-\rho}} \quad \text { for } t \in(a, T] .
$$

By the hypothesis $f(\cdot, x(\cdot)) \in C_{2-q, \rho}[a, T]$, we have $\left(\tau^{\rho}-a^{\rho}\right)^{2-q} f(\tau, x(\tau)) \in C[a, T]$. Therefore $\left|\left(\tau^{\rho}-a^{\rho}\right)^{2-q} f\right| \leq L$ (here $L$ is a positive constant) and

$$
\begin{aligned}
\left|\int_{a}^{t} \frac{f d \tau}{\tau^{1-\rho}}\right| & \leq \int_{a}^{t}\left|\left(\tau^{\rho}-a^{\rho}\right)^{q-2}\left[\left(\tau^{\rho}-a^{\rho}\right)^{2-q} f\right]\right| \frac{d \tau^{\rho}}{\rho} \\
& \leq \frac{L\left(t^{\rho}-a^{\rho}\right)^{q-1}}{(q-1) \rho}
\end{aligned}
$$

and

$$
\begin{aligned}
\left|\int_{a}^{t} \frac{t^{\rho}-\tau^{\rho}}{\rho} \frac{f d \tau}{\tau^{1-\rho}}\right| & \leq \int_{a}^{t}\left|\frac{t^{\rho}-\tau^{\rho}}{\rho}\left[\frac{\tau^{\rho}-a^{\rho}}{\rho}\right]^{q-2}\left[\left(\tau^{\rho}-a^{\rho}\right)^{2-q} f\right]\right| \frac{d \tau^{\rho}}{\rho^{3-q}} \\
& \leq \frac{L B(2, q-1)}{\rho^{2-q}}\left[\frac{t^{\rho}-a^{\rho}}{\rho}\right]^{q}
\end{aligned}
$$

Thus $\left.{ }_{a}^{K} \mathcal{D}_{t}^{q-1, \rho} x(t)\right|_{t \rightarrow a+}=x_{1}$ and $\left.{ }_{a}^{K} \mathcal{I}_{t}^{2-q, \rho} x(t)\right|_{t \rightarrow a+}=x_{2}$. The proof is completed. 


\section{Some properties of (1.1)-(1.3)}

In this section, we give some properties of three impulsive systems (1.1)-(1.3):

(i)

$$
\begin{aligned}
& \lim _{\substack{J_{k}\left(x\left(t_{k}-\right)\right) \rightarrow 0 \text { for all } \\
\bar{J}_{l}\left(x\left(\bar{t}_{l}-\right)\right) \rightarrow 0 \text { for all } l \in\{1,2, \ldots, m\}}}\{\text { system }(1.1)\} \\
& =\lim _{J_{k}\left(x\left(t_{k}-\right)\right) \rightarrow 0 \text { for all } k \in\{1,2, \ldots, m\}}\{\operatorname{system}(1.2)\} \\
& =\lim _{\bar{J}_{l}\left(x\left(\bar{t}_{l}-\right)\right) \rightarrow 0 \text { for all } l \in\{1,2, \ldots, n\}}\{\operatorname{system}(1.3)\} \\
& =\left\{\begin{array}{l}
{ }_{a}^{K} \mathcal{D}_{t}^{q, \rho} x(t)=f(t, x(t)), \quad t \in\left(t_{0}, T\right], \\
\left.{ }_{t_{0}} \mathcal{D}_{t}^{q-1, \rho} x(t)\right|_{t \rightarrow t_{0^{+}}}=x_{1},\left.\quad{ }_{t_{0}} \mathcal{I}_{t}^{2-q, \rho} x(t)\right|_{t \rightarrow t_{0^{+}}}=x_{2} .
\end{array}\right. \\
& \Leftrightarrow \quad x(t)=\frac{x_{2}}{\Gamma(q-1)}\left[\frac{t^{\rho}-\left(t_{0}\right)^{\rho}}{\rho}\right]^{q-2}+\frac{x_{1}}{\Gamma(q)}\left[\frac{t^{\rho}-\left(t_{0}\right)^{\rho}}{\rho}\right]^{q-1} \\
& +\frac{1}{\Gamma(q)} \int_{t_{0}}^{t}\left[\frac{t^{\rho}-\tau^{\rho}}{\rho}\right]^{q-1} \frac{f d \tau}{\tau^{1-\rho}}
\end{aligned}
$$

for $t \in\left(t_{0}, T\right]$ and $f=f(\tau, x(\tau))$.

(ii) $\lim _{\bar{J}_{l}\left(x\left(\bar{t}_{l}-\right)\right) \rightarrow 0 \text { for all } l \in\{1,2, \ldots, n\}}\{$ system $(1.1)\}=\{\operatorname{system}(1.2)\}$.

(iii) $\lim _{J_{k}\left(x\left(t_{k}-\right)\right) \rightarrow 0 \text { for all } k \in\{1,2, \ldots, m\}}\{$ system $(1.1)\}=\{$ system $(1.3)\}$.

(iv)

$$
\begin{aligned}
& \lim _{\substack{t_{k} \rightarrow t_{p} \text { for all } k \in\{1,2, \ldots, m\} \\
\bar{t}_{l} \rightarrow \bar{t}_{r} \text { for all } l \in\{1,2, \ldots, n\} \text { and } \forall p \in\{1,2, \ldots, m\}, \forall r \in\{1,2, \ldots, n\}}}\{\text { system (1.1) } \\
& =\left\{\begin{array}{l}
{ }_{t_{0}}^{K} \mathcal{D}_{t}^{q, \rho} x(t)=f(t, x(t)), \quad t \in\left(t_{0}, T\right], t \neq t_{p} \text { and } t \neq \bar{t}_{r}, \\
{ }_{t_{0}} \mathcal{D}_{t_{p^{+}}}^{q-1, \rho} x\left(t_{p^{+}}\right)-{ }_{t_{0}}^{K} \mathcal{D}_{t_{p^{-}}}^{q-1, \rho} x\left(t_{p^{-}}\right)=\sum_{k=1}^{m} J_{k}\left(x\left(t_{p}-\right)\right), \\
{ }_{t_{0}} \mathcal{I}_{\bar{t}_{r+}}^{2-q, \rho} x\left(\bar{t}_{r}+\right)-{ }_{t_{0}}^{K} \mathcal{I}_{\bar{t}_{r-}}^{2-q, \rho} x\left(\bar{t}_{r}-\right)=\sum_{l=1}^{n} \bar{J}_{l}\left(x\left(\bar{t}_{r}-\right)\right), \\
\left.{ }_{t_{0}}^{K} \mathcal{D}_{t}^{q-1, \rho} x(t)\right|_{t \rightarrow t_{0^{+}}}=x_{1},\left.\quad{ }_{t_{0}}^{K} \mathcal{I}_{t}^{2-q, \rho} x(t)\right|_{t \rightarrow t_{0^{+}}}=x_{2} .
\end{array}\right.
\end{aligned}
$$

(v)

$$
\begin{aligned}
& \lim _{t_{k} \rightarrow t_{p} \text { for all } k \in\{1,2, \ldots, m\} \text { and } \forall p \in\{1,2, \ldots, m\}}\{\text { system }(1.2)\} \\
& =\left\{\begin{array}{l}
{ }_{t_{0}} \mathcal{D}_{t}^{q, \rho} x(t)=f(t, x(t)), \quad t \in\left(t_{0}, T\right] \text { and } t \neq t_{p}, \\
K_{t_{0}} \mathcal{D}_{t_{p^{+}}}^{q-1, \rho} x\left(t_{p^{+}}\right)-{ }_{t_{0}}^{K} \mathcal{D}_{t_{p^{-}}}^{q-1, \rho} x\left(t_{p^{-}}\right)=\sum_{k=1}^{m} J_{k}\left(x\left(t_{p^{-}}\right)\right), \\
\left.{ }_{t_{0}}^{K} \mathcal{D}_{t}^{q-1, \rho} x(t)\right|_{t \rightarrow t_{0+}}=x_{1},\left.\quad{ }_{t_{0}}^{K} \mathcal{I}_{t}^{2-q, \rho} x(t)\right|_{t \rightarrow t_{0}+}=x_{2} .
\end{array}\right.
\end{aligned}
$$

(vi) $\lim _{\bar{t}_{l} \rightarrow \bar{t}_{r} \text { for all } l \in\{1,2, \ldots, n\} \text { and } \forall r \in\{1,2, \ldots, n\}}\{\operatorname{system}(1.3)\}$

$$
=\left\{\begin{array}{l}
{ }_{t_{0}}^{K} \mathcal{D}_{t}^{q, \rho} x(t)=f(t, x(t)), \quad t \in\left(t_{0}, T\right], t \neq \bar{t}_{r}, \\
{ }_{t_{0}} \mathcal{I}_{\bar{t}_{r}+}^{2-q, \rho} x\left(\bar{t}_{r}+\right)-{ }_{t_{0}}^{K} \mathcal{I}_{\bar{t}_{r-}-}^{2-q, \rho} x\left(\bar{t}_{r}-\right)=\sum_{l=1}^{n} \bar{J}_{l}\left(x\left(\bar{t}_{r}-\right)\right), \\
\left.{ }_{t_{0}} \mathcal{D}_{t}^{q-1, \rho} x(t)\right|_{t \rightarrow t_{0^{+}}}=x_{1},\left.\quad{ }_{t_{0}}^{K} \mathcal{I}_{t}^{2-q, \rho} x(t)\right|_{t \rightarrow t_{0^{+}}}=x_{2} .
\end{array}\right.
$$


4 The equivalent integral equations of (1.1)-(1.4)

For simplicity, let $f=f(\tau, x(\tau))$ and

$$
\begin{aligned}
y(\ell, t)= & \frac{x_{2}+x_{1} \frac{\ell^{\rho}-\left(t_{0}\right)^{\rho}}{\rho}+\int_{t_{0}}^{\ell} \frac{\ell^{\rho}-\tau^{\rho}}{\rho} \frac{f d \tau}{\tau^{1-\rho}}}{\Gamma(q-1)}\left[\frac{t^{\rho}-\ell^{\rho}}{\rho}\right]^{q-2}+\frac{x_{1}+\int_{t_{0}}^{\ell} \frac{f d \tau}{\tau^{1-\rho}}}{\Gamma(q)}\left[\frac{t^{\rho}-\ell^{\rho}}{\rho}\right]^{q-1} \\
& +\frac{1}{\Gamma(q)} \int_{\ell}^{t}\left[\frac{t^{\rho}-\tau^{\rho}}{\rho}\right]^{q-1} \frac{f d \tau}{\tau^{1-\rho}}
\end{aligned}
$$

here $\ell \in\left\{t_{0}, t_{1}, t_{2}, \ldots, t_{m}, \bar{t}_{1}, \bar{t}_{2}, \ldots, \bar{t}_{n}, T\right\}$.

For $1<q \leq 2$, define some function spaces:

$$
\begin{aligned}
& \hat{C}_{2-q, \rho}\left[t_{0}, T\right]=\left\{x:\left(t_{0}, T\right] \rightarrow \mathbb{R}:\left[t^{\rho}-\left(t_{i}^{\prime}\right)^{\rho}\right]^{2-q} x(t) \in C\left[t_{i}^{\prime}, t_{i+1}^{\prime}\right], i=0,1, \ldots, M\right\} \\
& (\rho \neq 0), \\
& \hat{C}_{2-q, \rho}\left[t_{0}, T\right]=\left\{x:\left(t_{0}, T\right] \rightarrow \mathbb{R}:\left[\ln t-\ln \left(t_{i}^{\prime}\right)\right]^{2-q} x(t) \in C\left[t_{i}^{\prime}, t_{i+1}^{\prime}\right], i=0,1, \ldots, M\right\} \\
& (\rho=0) \text {, } \\
& \hat{C}_{2-q, \rho}^{q}\left[t_{0}, T\right]=\left\{x(t) \in \hat{C}_{2-q, \rho}\left[t_{0}, T\right]:{ }_{t_{0}}^{K} \mathcal{D}_{t}^{q, \rho} x(t) \in \hat{C}_{2-q, \rho}\left[t_{0}, T\right]\right\}, \\
& I C\left(\left[t_{0}, T\right], \mathbb{R}\right)=\left\{x(t) \in \hat{C}_{2-q, \rho}\left[t_{0}, T\right]:\right. \\
& { }_{t_{0}}^{K} \mathcal{D}_{t_{k^{-}}}^{q-1, \rho} x\left(t_{k}-\right)=\lim _{t \rightarrow t_{k^{-}}}{ }_{t_{0}}^{K} \mathcal{D}_{t}^{q-1, \rho} x(t)={ }_{t_{0}}^{K} \mathcal{D}_{t_{k}}^{q-1, \rho} x\left(t_{k}\right)<\infty \\
& \text { and }{ }_{t_{0}}^{K} \mathcal{D}_{t_{k^{+}}}^{q-1, \rho} x\left(t_{k}+\right)=\lim _{t \rightarrow t_{k^{+}}{ }_{t_{0}}} \mathcal{D}_{t}^{q-1, \rho} x(t)<\infty(\text { here } k=1,2, \ldots, m) \text {, } \\
& \text { and }{ }_{t_{0}}^{K} \mathcal{I}_{\bar{t}_{l^{-}}}^{2-q, \rho} x\left(\bar{t}_{l^{-}}\right)=\lim _{t \rightarrow \bar{t}_{l^{-}}}{ }_{t_{0}}^{K} \mathcal{I}_{t}^{2-q, \rho} x(t)={ }_{t_{0}}^{K} \mathcal{I}_{\bar{t}_{l}}^{2-q, \rho} x\left(\bar{t}_{l}\right)<\infty \text { and } \\
& \left.{ }_{t_{0}}^{K} \mathcal{I}_{\bar{t}_{l^{+}}}^{2-q, \rho} x\left(\bar{t}_{l}+\right)=\lim _{t \rightarrow \bar{t}_{l^{+}}}{ }_{t_{0}} \mathcal{I}_{t}^{2-q, \rho} x(t)<\infty(\text { here } l=1,2, \ldots, n)\right\} ; \\
& \tilde{C}_{2-q, \rho}\left[t_{0}, T\right]=\left\{x:\left(t_{0}, T\right] \rightarrow \mathbb{R}:\left[t^{\rho}-\left(t_{i}\right)^{\rho}\right]^{2-q} x(t) \in C\left[t_{i}, t_{i+1}\right], i=0,1, \ldots, m\right\} \\
& (\rho \neq 0) \text {, } \\
& \tilde{C}_{2-q, \rho}\left[t_{0}, T\right]=\left\{x:\left(t_{0}, T\right] \rightarrow \mathbb{R}:\left[\ln t-\ln \left(t_{i}\right)\right]^{2-q} x(t) \in C\left[t_{i}, t_{i+1}\right], i=0,1, \ldots, m\right\} \\
& (\rho=0), \\
& \tilde{C}_{2-q, \rho}^{q}\left[t_{0}, T\right]=\left\{x(t) \in \tilde{C}_{2-q, \rho}\left[t_{0}, T\right]:{ }_{t_{0}}^{K} \mathcal{D}_{t}^{q, \rho} x(t) \in \tilde{C}_{2-q, \rho}\left[t_{0}, T\right]\right\}, \\
& I C_{1}\left(\left[t_{0}, T\right], \mathbb{R}\right)=\left\{x(t) \in \tilde{C}_{2-q, \rho}\left[t_{0}, T\right]:{ }_{t_{0}}^{K} \mathcal{D}_{t_{k^{+}}}^{q-1, \rho} x\left(t_{k}+\right)=\lim _{t \rightarrow t_{k}+}{ }_{t_{0}}^{K} \mathcal{D}_{t}^{q-1, \rho} x(t)<\infty,\right. \\
& { }_{t_{0}}^{K} \mathcal{D}_{t_{k^{-}}}^{q-1, \rho} x\left(t_{k^{-}}\right)=\lim _{t \rightarrow t_{k^{-}}}{ }_{t_{0}}^{K} \mathcal{D}_{t}^{q-1, \rho} x(t)={ }_{t_{0}}^{K} \mathcal{D}_{t_{k}}^{q-1, \rho} x\left(t_{k}\right)<\infty, \\
& \text { and } \left.{ }_{t_{0}}^{K} \mathcal{I}_{t_{k^{+}}}^{2-q, \rho} x\left(t_{k}+\right)={ }_{t_{0}}^{K} \mathcal{I}_{t_{k^{-}}}^{2-q, \rho} x\left(t_{k^{-}}\right) \text {, here } k=1,2, \ldots, m\right\} \text {; } \\
& \bar{C}_{2-q, \rho}\left[t_{0}, T\right]=\left\{x:\left(t_{0}, T\right] \rightarrow \mathbb{R}:\left[t^{\rho}-\left(\bar{t}_{j}\right)^{\rho}\right]^{2-q} x(t) \in C\left[\bar{t}_{j}, \bar{t}_{j+1}\right], j=0,1, \ldots, n\right\} \\
& (\rho \neq 0), \\
& \bar{C}_{2-q, \rho}\left[t_{0}, T\right]=\left\{x:\left(t_{0}, T\right] \rightarrow \mathbb{R}:\left[\ln t-\ln \left(\bar{t}_{j}\right)\right]^{2-q} x(t) \in C\left[\bar{t}_{j}, \bar{t}_{j+1}\right], j=0,1, \ldots, n\right\} \\
& (\rho=0),
\end{aligned}
$$




$$
\begin{aligned}
\bar{C}_{2-q, \rho}^{q}\left[t_{0}, T\right]=\{ & \left.x(t) \in \bar{C}_{2-q, \rho}\left[t_{0}, T\right]:{ }_{t_{0}}^{K} \mathcal{D}_{t}^{q, \rho} x(t) \in \bar{C}_{2-q, \rho}\left[t_{0}, T\right]\right\}, \\
I C_{2}\left(\left[t_{0}, T\right], \mathbb{R}\right)= & \left\{x(t) \in \bar{C}_{2-q, \rho}\left[t_{0}, T\right]:{ }_{t_{0}}^{K} \mathcal{I}_{\bar{t}_{l^{+}}}^{2-q, \rho} x\left(\bar{t}_{l}+\right)=\lim _{t \rightarrow \bar{t}_{l^{+}}}{ }_{t_{0}}^{K} \mathcal{I}_{t}^{2-q, \rho} x(t)<\infty,\right. \\
& { }_{t_{0}}^{K} \mathcal{I}_{\bar{t}_{l^{-}}}^{2-q, \rho} x\left(\bar{t}_{l^{-}}\right)=\lim _{t \rightarrow \bar{t}_{l^{-}}}{ }_{t_{0}}^{K} \mathcal{I}_{t}^{2-q, \rho} x(t)={ }_{t_{0}}^{K} \mathcal{I}_{\bar{t}_{l}}^{2-q, \rho} x\left(\bar{t}_{l}\right)<\infty \\
& \text { and } \left.{ }_{t_{0}}^{K} \mathcal{D}_{\bar{t}_{l^{+}}}^{q-1, \rho} x\left(\bar{t}_{l+}\right)={ }_{t_{0}}^{K} \mathcal{D}_{\bar{t}_{l^{-}}}^{q-1, \rho} x\left(\bar{t}_{l}-\right), \text { here } l=1,2, \ldots, n\right\} .
\end{aligned}
$$

Next, we seek the equivalent integral equation of (1.2). Considering ${ }_{t_{0}}^{K} \mathcal{D}_{t}^{q, \rho} x(t)=f(t, x(t))$ on each piecewise interval $\left(t_{k}, t_{k+1}\right](k=1,2, \ldots, m)$ by Lemma 2.4 , we find a piecewise function

$$
\tilde{x}(t)=\left\{\begin{array}{l}
y\left(t_{0}, t\right) \quad \text { for } t \in\left(t_{0}, t_{1}\right], \\
\frac{K \mathcal{I}_{t_{k}}^{2-q, \rho} x\left(t_{k}+\right)}{\Gamma(q-1)}\left[\frac{t^{\rho}-\left(t_{k}\right)^{\rho}}{\rho}\right]^{q-2}+\frac{K_{0} \mathcal{D}_{t_{k}}^{q-1, \rho} x\left(t_{k}+\right)}{\Gamma(q)}\left[\frac{t^{\rho}-\left(t_{k}\right)^{\rho}}{\rho}\right]^{q-1} \\
\quad+\frac{1}{\Gamma(q)} \int_{t_{k}}^{t}\left[\frac{t^{\rho}-\tau^{\rho}}{\rho}\right]^{q-1} \frac{f d \tau}{\tau^{1-\rho}} \quad \text { for } t \in\left(t_{k}, t_{k+1}\right], k=1,2, \ldots, m,
\end{array}\right.
$$

with ${ }_{t_{0}}^{K} \mathcal{D}_{t_{k^{+}}}^{q-1, \rho} x\left(t_{k}+\right)={ }_{t_{0}}^{K} \mathcal{D}_{t_{k^{-}}}^{q-1, \rho} x\left(t_{k}-\right)+J_{k}\left(x\left(t_{k}-\right)\right)$ and ${ }_{t_{0}}^{K} \mathcal{I}_{t_{k^{+}}}^{2-q, \rho} x\left(t_{k^{+}}+\right)={ }_{t_{0}}^{K} \mathcal{I}_{t_{k^{-}}}^{2-q, \rho} x\left(t_{k}-\right)$.

Because (4.2) does not satisfy property (i), $\tilde{x}(t)$ is only considered as an approximate solution of (1.2). And let

$$
e_{k}(t)=x(t)-\tilde{x}(t), \quad \text { for } t \in\left(t_{k}, t_{k+1}\right](k=1,2, \ldots, m) \text {, }
$$

where $x(t)$ represents the exact solution of (1.2).

Lemma 4.1 Let $q \in(1,2]$ and $t_{0}, \rho>0$, and let $f:\left[t_{0}, T\right] \times \mathbb{R} \rightarrow \mathbb{R}$ be a function such that $f(\cdot, x(\cdot)) \in \tilde{C}_{2-q, \rho}\left[t_{0}, T\right]$ for any $x(\cdot) \in \tilde{C}_{2-q, \rho}\left[t_{0}, T\right]$.

If $x(\cdot) \in I C_{1}\left(\left[t_{0}, T\right], \mathbb{R}\right)$, then $x(t)$ is a solution of $(1.2)$ if, and only if, $x(t)$ satisfies

$$
x(t)=\left\{\begin{array}{l}
y\left(t_{0}, t\right) \quad \text { for } t \in\left(t_{0}, t_{1}\right], \\
y\left(t_{0}, t\right)+\sum_{i=1}^{k} \frac{J_{i}\left(x\left(t_{i}-\right)\right)}{\Gamma(q)}\left[\frac{t^{\rho}-\left(t_{i}\right)^{\rho}}{\rho}\right]^{q-1}+\xi \sum_{i=1}^{k} J_{i}\left(x\left(t_{i}-\right)\right)\left[y\left(t_{i}, t\right)-y\left(t_{0}, t\right)\right] \\
\quad \text { for } t \in\left(t_{k}, t_{k+1}\right], k=1,2, \ldots, m,
\end{array}\right.
$$

where $\xi$ is an arbitrary constant.

Proof First, we prove the necessity by applying mathematical induction. By Lemma 2.4, the solution of (1.2) as $t \in\left(t_{0}, t_{1}\right]$ satisfies

$$
x(t)=y\left(t_{0}, t\right) \quad \text { for } t \in\left(t_{0}, t_{1}\right] .
$$

Using two operators ${ }_{t_{0}}^{K} \mathcal{D}_{t}^{q-1, \rho}$ and ${ }_{t_{0}}^{K} \mathcal{I}_{t}^{2-q, \rho}$ to two sides of (4.5), respectively, we have

$$
\begin{aligned}
{ }_{t_{0}}^{K} \mathcal{D}_{t_{1+}}^{q-1, \rho} x\left(t_{1}+\right) & ={ }_{t_{0}}^{K} \mathcal{D}_{t_{1-}}^{q-1, \rho} x\left(t_{1}-\right)+J_{1}\left(x\left(t_{1}-\right)\right) \\
& =x_{1}+\int_{t_{0}}^{t_{1}} \frac{f d \tau}{\tau^{1-\rho}}+J_{1}\left(x\left(t_{1}-\right)\right)
\end{aligned}
$$


and

$$
\begin{aligned}
{ }_{t_{0}}^{K} \mathcal{I}_{t_{1+}+}^{2-q, \rho} x\left(t_{1}+\right) & ={ }_{t_{0}}^{K} \mathcal{I}_{t_{1-}-}^{2-q, \rho} x\left(t_{1}-\right) \\
& =x_{2}+x_{1} \frac{\left(t_{1}\right)^{\rho}-\left(t_{0}\right)^{\rho}}{\rho}+\int_{t_{0}}^{t_{1}} \frac{\left(t_{1}\right)^{\rho}-\tau^{\rho}}{\rho} \frac{f d \tau}{\tau^{1-\rho}} .
\end{aligned}
$$

Substituting (4.6)-(4.7) into (4.2), the approximate solution of (1.2) as $t \in\left(t_{1}, t_{2}\right]$ is given as

$$
\tilde{x}(t)=y\left(t_{1}, t\right)+\frac{J_{1}\left(x\left(t_{1}-\right)\right)}{\Gamma(q)}\left[\frac{t^{\rho}-\left(t_{1}\right)^{\rho}}{\rho}\right]^{q-1} \text { for } t \in\left(t_{1}, t_{2}\right] .
$$

By (4.5) the exact solution of (1.2) as $t \in\left(t_{1}, t_{2}\right]$ satisfies

$$
\lim _{J_{1}\left(x\left(t_{1}-\right)\right) \rightarrow 0} x(t)=y\left(t_{0}, t\right) \quad \text { for } t \in\left(t_{1}, t_{2}\right] .
$$

By (4.3) and (4.8)-(4.9), we get

$$
\lim _{J_{1}\left(x\left(t_{1}-\right)\right) \rightarrow 0} e_{1}(t)=y\left(t_{0}, t\right)-y\left(t_{1}, t\right) \quad \text { for } t \in\left(t_{1}, t_{2}\right]
$$

From (4.10), let $e_{1}(t)=\kappa\left(J_{1}\left(x\left(t_{1}^{-}\right)\right)\right) \lim _{J_{1}\left(x\left(t_{1}^{-}\right)\right) \rightarrow 0} e_{1}(t)$, where $\kappa(\cdot)$ is an undetermined function to satisfy $\kappa(0)=1$, and

$$
e_{1}(t)=\kappa\left(J_{1}\left(x\left(t_{1}-\right)\right)\right) \lim _{J_{1}\left(x\left(t_{1}-\right)\right) \rightarrow 0} e_{1}(t)=-\kappa\left(J_{1}\left(x\left(t_{1}-\right)\right)\right)\left[y\left(t_{1}, t\right)-y\left(t_{0}, t\right)\right] .
$$

Plugging (4.8) and (4.11) into (4.3), we obtain

$$
\begin{aligned}
& x(t)=y\left(t_{0}, t\right)+\frac{J_{1}\left(x\left(t_{1}-\right)\right)}{\Gamma(q)}\left[\frac{t^{\rho}-\left(t_{1}\right)^{\rho}}{\rho}\right]^{q-1}+\left[1-\kappa\left(J_{1}\left(x\left(t_{1}-\right)\right)\right)\right]\left[y\left(t_{1}, t\right)-y\left(t_{0}, t\right)\right] \\
& \quad \text { for } t \in\left(t_{1}, t_{2}\right] .
\end{aligned}
$$

Because ${ }_{t_{0}}^{K} \mathcal{D}_{t}^{q, 0+}\left({ }_{t_{0}}^{K} \mathcal{D}_{t}^{q, \rho}\right.$ with $\left.\rho \rightarrow 0+\right)$ is the Hadamard fractional derivative, we get $1-$ $\kappa\left(J_{1}\left(x\left(t_{1}^{-}\right)\right)\right)=\xi J_{1}\left(x\left(t_{1}^{-}\right)\right)$(here $\xi$ is an arbitrary constant) by applying Lemma 3.3 in [44] to (1.2) and (4.12) with $\rho \rightarrow 0+$. Thus (4.12) is rewritten as

$$
\begin{aligned}
& x(t)=y\left(t_{0}, t\right)+\frac{J_{1}\left(x\left(t_{1}-\right)\right)}{\Gamma(q)}\left[\frac{t^{\rho}-\left(t_{1}\right)^{\rho}}{\rho}\right]^{q-1}+\xi J_{1}\left(x\left(t_{1}-\right)\right)\left[y\left(t_{1}, t\right)-y\left(t_{0}, t\right)\right] \\
& \quad \text { for } t \in\left(t_{1}, t_{2}\right] .
\end{aligned}
$$

Therefore the solution of (1.2) satisfies (4.4) as $t \in\left(t_{1}, t_{2}\right]$.

For $t \in\left(t_{k}, t_{k+1}\right]$, suppose that the solution of (1.2) satisfies

$$
x(t)=y\left(t_{0}, t\right)+\sum_{i=1}^{k} \frac{J_{i}\left(x\left(t_{i}-\right)\right)}{\Gamma(q)}\left[\frac{t^{\rho}-\left(t_{i}\right)^{\rho}}{\rho}\right]^{q-1}+\xi \sum_{i=1}^{k} J_{i}\left(x\left(t_{i}-\right)\right)\left[y\left(t_{i}, t\right)-y\left(t_{0}, t\right)\right]
$$

$$
\text { for } t \in\left(t_{k}, t_{k+1}\right]
$$

to prove that the solution of (1.2) satisfies (4.4) as $t \in\left(t_{k+1}, t_{k+2}\right]$. 
Using operators ${ }_{t_{0}}^{K} \mathcal{D}_{t}^{q-1, \rho}$ and ${ }_{t_{0}}^{K} \mathcal{I}_{t}^{2-q, \rho}$ to two sides of (4.14) respectively, we obtain

$$
\begin{aligned}
{ }_{t_{0}}^{K} \mathcal{D}_{t_{k+1}+}^{q-1, \rho} x\left(t_{k+1}+\right) & ={ }_{t_{0}}^{K} \mathcal{D}_{t_{k+1}-}^{q-1, \rho} x\left(t_{k+1}-\right)+J_{k+1}\left(x\left(t_{k+1}-\right)\right) \\
& =x_{1}+\int_{t_{0}}^{t_{k+1}} \frac{f d \tau}{\tau^{1-\rho}}+\sum_{i=1}^{k+1} J_{i}\left(x\left(t_{i}-\right)\right)
\end{aligned}
$$

and

$$
\begin{aligned}
{ }_{t_{0}}^{K} \mathcal{I}_{t_{k+1}+}^{2-q, \rho} x\left(t_{k+1}+\right)= & { }_{t_{0}}^{K} \mathcal{I}_{t_{k+1}-}^{2-q, \rho} x\left(t_{k+1}-\right) \\
= & x_{2}+x_{1} \frac{\left(t_{k+1}\right)^{\rho}-\left(t_{0}\right)^{\rho}}{\rho}+\int_{t_{0}}^{t_{k+1}} \frac{\left(t_{k+1}\right)^{\rho}-\tau^{\rho}}{\rho} \frac{f d \tau}{\tau^{1-\rho}} \\
& +\sum_{i=1}^{k} J_{i}\left(x\left(t_{i}-\right)\right) \frac{\left(t_{k+1}\right)^{\rho}-\left(t_{i}\right)^{\rho}}{\rho} .
\end{aligned}
$$

Plugging (4.15) and (4.16) into (4.2), the approximate solution of (1.2) as $t \in\left(t_{k+1}, t_{k+2}\right]$ is given by

$$
\begin{aligned}
\tilde{x}(t)= & y\left(t_{k+1}, t\right)+\frac{\sum_{i=1}^{k} J_{i}\left(x\left(t_{i}-\right)\right) \frac{\left(t_{k+1}\right)^{\rho}-\left(t_{i}\right)^{\rho}}{\rho}}{\Gamma(q-1)}\left[\frac{t^{\rho}-\left(t_{k+1}\right)^{\rho}}{\rho}\right]^{q-2} \\
& +\frac{\sum_{i=1}^{k+1} J_{i}\left(x\left(t_{i}-\right)\right)}{\Gamma(q)}\left[\frac{t^{\rho}-\left(t_{k+1}\right)^{\rho}}{\rho}\right]^{q-1} \text { for } t \in\left(t_{k+1}, t_{k+2}\right] .
\end{aligned}
$$

On the other hand, by (4.14) the exact solution of (1.2) as $t \in\left(t_{k+1}, t_{k+2}\right]$ satisfies

$$
\lim _{J_{i}\left(x\left(t_{i}-\right)\right) \rightarrow 0 \text { for all }} x(t)=y\left(t_{0}, t\right) \quad \text { for } t \in\left(t_{k+1}, t_{k+2}\right]
$$

and

$$
\begin{aligned}
\lim _{\substack{p\left(x\left(t_{p}-\right)\right) \rightarrow 0 \text { here } \\
p \in\{1,2, \ldots, k+1\}}} x(t)= & y\left(t_{0}, t\right)+\sum_{\substack{1 \leq i \leq k+1 \\
\text { and } i \neq p}} \frac{J_{i}\left(x\left(t_{i}-\right)\right)}{\Gamma(q)}\left[\frac{t^{\rho}-\left(t_{i}\right)^{\rho}}{\rho}\right]^{q-1} \\
& +\xi \sum_{\substack{1 \leq i \leq k+1 \\
\text { and } i \neq p}} J_{i}\left(x\left(t_{i}-\right)\right)\left[y\left(t_{i}, t\right)-y\left(t_{0}, t\right)\right] \quad \text { for } t \in\left(t_{k+1}, t_{k+2}\right] .
\end{aligned}
$$

By (4.3) and (4.17)-(4.19), we have

$$
\lim _{\substack{J_{i}\left(x\left(t_{i}-\right)\right) \rightarrow 0 \text { for } \\ \text { all } i \in\{1,2, \ldots, k+1\}}} e_{k+1}(t)=\lim _{\substack{J_{i}\left(x\left(t_{i}-\right)\right) \rightarrow 0 \text { for } \\ \text { all } i \in\{1,2, \ldots, k+1\}}}\{x(t)-\tilde{x}(t)\}=-\left[y\left(t_{k+1}, t\right)-y\left(t_{0}, t\right)\right]
$$

and

$$
\begin{aligned}
\lim _{\substack{J_{p}\left(x\left(t_{p}-\right)\right) \rightarrow 0 \text { here } \\
p \in\{1,2, \ldots, k+1\}}} e_{k+1}(t) & =\lim _{\substack{J_{p}\left(x\left(t_{p}-\right)\right) \rightarrow 0 \text { here } \\
p \in\{1,2, \ldots, k+1\}}}\{x(t)-\tilde{x}(t)\} \\
& =-\left[y\left(t_{k+1}, t\right)-y\left(t_{0}, t\right)\right]+\xi \sum_{\substack{1 \leq i \leq k+1 \\
\text { and } i \neq p}} J_{i}\left(x\left(t_{i}-\right)\right)\left[y\left(t_{i}, t\right)-y\left(t_{0}, t\right)\right]
\end{aligned}
$$




$$
\begin{aligned}
& +\sum_{\substack{1 \leq i \leq k+1 \\
\text { and } i \neq p}} \frac{J_{i}\left(x\left(t_{i}-\right)\right)}{\Gamma(q)}\left\{\left[\frac{t^{\rho}-\left(t_{i}\right)^{\rho}}{\rho}\right]^{q-1}-\left[\frac{t^{\rho}-\left(t_{k+1}\right)^{\rho}}{\rho}\right]^{q-1}\right\} \\
& -\sum_{\substack{1 \leq i \leq k+1 \\
\text { and } i \neq p}} \frac{J_{i}\left(x\left(t_{i}-\right)\right) \frac{\left(t_{k+1}\right)^{\rho}-\left(t_{i}\right)^{\rho}}{\rho}}{\Gamma(q-1)}\left[\frac{t^{\rho}-\left(t_{k+1}\right)^{\rho}}{\rho}\right]^{q-2} .
\end{aligned}
$$

By (4.20) and (4.21), we obtain

$$
\begin{aligned}
e_{k+1}(t)= & -\left[y\left(t_{k+1}, t\right)-y\left(t_{0}, t\right)\right]+\xi \sum_{i=1}^{k+1} J_{i}\left(x\left(t_{i}-\right)\right)\left[y\left(t_{i}, t\right)-y\left(t_{0}, t\right)\right] \\
& +\sum_{i=1}^{k+1} \frac{J_{i}\left(x\left(t_{i}-\right)\right)}{\Gamma(q)}\left\{\left[\frac{t^{\rho}-\left(t_{i}\right)^{\rho}}{\rho}\right]^{q-1}-\left[\frac{t^{\rho}-\left(t_{k+1}\right)^{\rho}}{\rho}\right]^{q-1}\right\} \\
& -\sum_{i=1}^{k+1} \frac{J_{i}\left(x\left(t_{i}-\right)\right) \frac{\left(t_{k+1}\right)^{\rho}-\left(t_{i}\right)^{\rho}}{\rho}}{\Gamma(q-1)}\left[\frac{t^{\rho}-\left(t_{k+1}\right)^{\rho}}{\rho}\right]^{q-2} .
\end{aligned}
$$

Thus, substituting (4.17) and (4.22) into (4.3), we get

$$
\begin{aligned}
& x(t)=y\left(t_{0}, t\right)+\sum_{i=1}^{k+1} \frac{J_{i}\left(x\left(t_{i}-\right)\right)}{\Gamma(q)}\left[\frac{t^{\rho}-\left(t_{i}\right)^{\rho}}{\rho}\right]^{q-1}+\xi \sum_{i=1}^{k+1} J_{i}\left(x\left(t_{i}-\right)\right)\left[y\left(t_{i}, t\right)-y\left(t_{0}, t\right)\right] \\
& \quad \text { for } t \in\left(t_{k+1}, t_{k+2}\right] .
\end{aligned}
$$

Therefore the solution of (1.2) satisfies (4.4) as $t \in\left(t_{k+1}, t_{k+2}\right]$. Hence the necessity is proved.

Now we prove the sufficiency. Applying the operators ${ }_{t_{0}}^{K} \mathcal{D}_{t}^{q, \rho},{ }_{t_{0}}^{K} \mathcal{D}_{t}^{q-1, \rho}$, and ${ }_{t_{0}}^{K} \mathcal{I}_{t}^{2-q, \rho}$ to two sides of (4.4) as $t \in\left(t_{k}, t_{k+1}\right]$, respectively, we have

$$
\begin{aligned}
&\left.{ }_{t_{0}}^{K} \mathcal{D}_{t}^{q, \rho} x(t)\right|_{t \in\left(t_{k}, t_{k+1}\right]}\left\{\left.f(t, x(t))\right|_{t \geq t_{0}}+\xi \sum_{i=1}^{k} J_{i}\left(x\left(t_{i}-\right)\right)\left[\left.f(t, x(t))\right|_{t \geq t_{i}}-\left.f(t, x(t))\right|_{t \geq t_{0}}\right]\right\}_{t \in\left(t_{k}, t_{k+1}\right]} \\
&=\left.f(t, x(t))\right|_{t \in\left(t_{k}, t_{k+1}\right]} \\
&\left.{ }_{t_{0}}^{K} \mathcal{D}_{t}^{q-1, \rho} x(t)\right|_{t \in\left(t_{k}, t_{k+1}\right]} \\
&=\left\{x_{1}+\int_{t_{0}}^{t} \frac{f d \tau}{\tau^{1-\rho}}+\sum_{i=1}^{k} J_{i}\left(x\left(t_{i}-\right)\right)\right. \\
&\left.+\sum_{i=1}^{k} \xi J_{i}\left(x\left(t_{i}-\right)\right)\left[x_{1}+\int_{t_{0}}^{t_{i}} \frac{f d \tau}{\tau^{1-\rho}}+\int_{t_{i}}^{t} \frac{f d \tau}{\tau^{1-\rho}}-x_{1}-\int_{t_{0}}^{t} \frac{f d \tau}{\tau^{1-\rho}}\right]\right\}_{t \in\left(t_{k}, t_{k+1}\right]} \\
&= \\
& \quad\left\{x_{1}+\int_{t_{0}}^{t} \frac{f d \tau}{\tau^{1-\rho}}+\sum_{i=1}^{k} J_{i}\left(x\left(t_{i}-\right)\right)\right\} \\
& t \in\left(t_{k}, t_{k+1}\right]
\end{aligned}
$$


and

$$
\begin{aligned}
& \left.{ }_{t_{0}}^{K} \mathcal{I}_{t}^{2-q, \rho} x(t)\right|_{t \in\left(t_{k}, t_{k+1}\right]} \\
& \quad=\left\{x_{2}+x_{1} \frac{t^{\rho}-\left(t_{0}\right)^{\rho}}{\rho}+\int_{t_{0}}^{t} \frac{t^{\rho}-\tau^{\rho}}{\rho} \frac{f d \tau}{\tau^{1-\rho}}+\sum_{i=1}^{k} J_{i}\left(x\left(t_{i}-\right)\right) \frac{t^{\rho}-\left(t_{i}\right)^{\rho}}{\rho}\right\}_{t \in\left(t_{k}, t_{k+1}\right]} .
\end{aligned}
$$

Thus $\left.{ }_{t_{0}}^{K} \mathcal{D}_{t}^{q-1, \rho} x(t)\right|_{t \rightarrow t_{0}+}=x_{1},\left.{ }_{t_{0}}^{K} \mathcal{I}_{t}^{2-q, \rho} x(t)\right|_{t \rightarrow t_{0}{ }^{+}}=x_{2},{ }_{t_{0}}^{K} \mathcal{D}_{t_{k}{ }^{+}}^{q-1, \rho} x\left(t_{k}+\right)-{ }_{t_{0}}^{K} \mathcal{D}_{t_{k^{-}}}^{q-1, \rho} x\left(t_{k}-\right)=$ $J_{k}\left(x\left(t_{k}-\right)\right)$, and ${ }_{t_{0}}^{K} \mathcal{I}_{t_{k^{+}}}^{2-q, \rho} x\left(t_{k}+\right)={ }_{t_{0}}^{K} \mathcal{I}_{t_{k}-}^{2-q, \rho} x\left(t_{k}-\right)$, and (4.4) satisfies the condition of fractional derivative in (1.2).

Letting $J_{k}\left(x\left(t_{k}-\right)\right)=0$ for all $k \in\{1,2, \ldots, m\}$ in (4.3), we obtain

$$
\begin{aligned}
& \lim _{J_{k}\left(x\left(t_{k}-\right)\right) \rightarrow 0 \text { for all }}\{\text { Eq\{1,2,..,m\} }\{\text { Eq. (4.3) }\} \text { is equivalent to }
\end{aligned}
$$

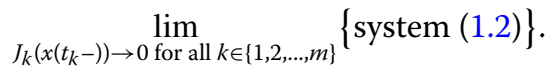

Moreover, it is obvious that (4.4) satisfies condition (v). Therefore (4.4) satisfies all the conditions of (1.2). Hence, this proof is completed.

Remark 4.2 Similar to (4.2), an approximate solution of (1.3) is presented by

$$
\tilde{\tilde{x}}(t)=\left\{\begin{array}{l}
y\left(t_{0}, t\right) \quad \text { for } t \in\left(t_{0}, \bar{t}_{1}\right], \\
\frac{K{ }_{t_{0}} \mathcal{I}_{\bar{t}_{l^{+}}-q, \rho}^{2-q\left(\bar{t}_{l}+\right)}}{\Gamma(q-1)}\left[\frac{t^{\rho}-\left(\bar{t}_{l}\right)^{\rho}}{\rho}\right]^{q-2}+\frac{K_{0} \mathcal{D}_{\bar{t}_{l^{+}}}^{q-1, \rho} x\left(\bar{t}_{l}+\right)}{\Gamma(q)}\left[\frac{t^{\rho}-\left(\bar{t}_{l}\right)^{\rho}}{\rho}\right]^{q-1} \\
\quad+\frac{1}{\Gamma(q)} \int_{\bar{t}_{l}}^{t}\left[\frac{t^{\rho}-\tau^{\rho}}{\rho}\right]^{q-1} \frac{f d \tau}{\tau^{1-\rho}} \quad \text { for } t \in\left(\bar{t}_{l}, \bar{t}_{l+1}\right], l=1,2, \ldots, n,
\end{array}\right.
$$

with ${ }_{t_{0}}^{K} \mathcal{I}_{\bar{t}_{l^{+}}}^{2-q, \rho} x\left(\bar{t}_{l^{+}}\right)={ }_{t_{0}}^{K} \mathcal{I}_{\bar{t}_{l^{-}}}^{2-q, \rho} x\left(\bar{t}_{l}-\right)+\bar{J}_{l}\left(x\left(\bar{t}_{l^{-}}\right)\right)$and ${ }_{t_{0}}^{K} \mathcal{D}_{\bar{t}_{l^{+}}}^{q-1, \rho} x\left(\bar{t}_{l^{+}}\right)={ }_{t_{0}}^{K} \mathcal{D}_{\bar{t}_{l^{-}}}^{q-1, \rho} x\left(\bar{t}_{l^{-}}\right)$.

Furthermore, using the thought of Lemma 4.1, we arrive at the following conclusion.

Lemma 4.3 Let $q \in(1,2]$ and $t_{0}, \rho>0$, and let $f:\left[t_{0}, T\right] \times \mathbb{R} \rightarrow \mathbb{R}$ be a function such that $f(\cdot, x(\cdot)) \in \bar{C}_{2-q, \rho}\left[t_{0}, T\right]$ for any $x(\cdot) \in \bar{C}_{2-q, \rho}\left[t_{0}, T\right]$.

If $x(\cdot) \in I C_{2}\left(\left[t_{0}, T\right], \mathbb{R}\right)$, then $x(t)$ is a solution of $(1.3)$ if, and only if, $x(t)$ satisfies the following integral equation:

$$
x(t)=\left\{\begin{array}{l}
y\left(t_{0}, t\right) \quad \text { for } t \in\left(t_{0}, \bar{t}_{1}\right], \\
y\left(t_{0}, t\right)+\sum_{j=1}^{l} \frac{\bar{J}_{j}\left(x\left(\bar{t}_{j}-\right)\right)}{\Gamma(q-1)}\left[\frac{t^{\rho}-\left(\bar{t}_{j}\right)^{\rho}}{\rho}\right]^{q-2}+\eta \sum_{j=1}^{l} \bar{J}_{j}\left(x\left(\bar{t}_{j}-\right)\right)\left[y\left(\bar{t}_{j}, t\right)-y\left(t_{0}, t\right)\right] \\
\quad \text { for } t \in\left(\bar{t}_{l}, \bar{t}_{l+1}\right], l=1,2, \ldots, n,
\end{array}\right.
$$

where $\eta$ is an arbitrary constant. 
The following theorem yields the equivalence between Cauchy problem (1.1) and the Volterra integral equation of the second kind:

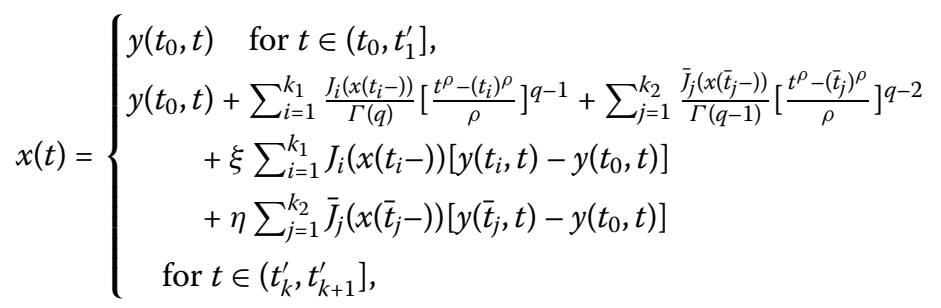

where $\xi$ and $\eta$ are two arbitrary constants.

Theorem 4.4 Let $q \in(1,2]$ and $t_{0}, \rho>0$, and let $f:[a, T] \times \mathbb{R} \rightarrow \mathbb{R}$ be a function such that $f(\cdot, x(\cdot)) \in \hat{C}_{2-q, \rho}\left[t_{0}, T\right]$ for any $x(\cdot) \in \hat{C}_{2-q, \rho}[a, T]$.

If $x(\cdot) \in I C\left(\left[t_{0}, T\right], \mathbb{R}\right)$, then $x(t)$ is a solution of $(1.1)$ if, and only if, $x(t)$ satisfies (4.25).

Proof First, we prove the necessity that the solution of (1.1) satisfies (4.25) by the mathematical induction. For $t \in\left(t_{0}, t_{1}^{\prime}\right]$, by Lemma 2.4, the solution of system (1.1) satisfies (4.25) and

$$
x(t)=y\left(t_{0}, t\right) \quad \text { for } t \in\left(t_{0}, t_{1}^{\prime}\right] .
$$

For $t \in\left(t_{1}^{\prime}, t_{2}^{\prime}\right]$, there appear three cases $t_{1}^{\prime}=t_{1}<\bar{t}_{1}, t_{1}^{\prime}=\bar{t}_{1}<t_{1}$, and $t_{1}^{\prime}=t_{1}=\bar{t}_{1}$. For $t_{1}^{\prime}=$ $t_{1}<\bar{t}_{1}$ and $t_{1}^{\prime}=\bar{t}_{1}<t_{1}$, the solution of (1.1) satisfies (4.25) as $t \in\left(t_{1}^{\prime}, t_{2}^{\prime}\right]$ by Lemmas 4.1 and 4.3, respectively. Hence, we need only prove that the solution of (1.1) satisfies (4.25) as $t \in\left(t_{1}^{\prime}, t_{2}^{\prime}\right]$ with $t_{1}^{\prime}=t_{1}=\bar{t}_{1}$. Applying ${ }_{t_{0}}^{K} \mathcal{D}_{t}^{q-1, \rho}$ and ${ }_{t_{0}}^{K} \mathcal{I}_{t}^{2-q, \rho}$ to two sides of (4.26), we have

$$
{ }_{t_{0}}^{K} \mathcal{D}_{t_{1}^{\prime}+}^{q-1, \rho} x\left(t_{1}^{\prime}+\right)={ }_{t_{0}}^{K} \mathcal{D}_{t_{1}^{\prime}-}^{q-1, \rho} x\left(t_{1}^{\prime}-\right)+J_{1}\left(x\left(t_{1}^{\prime}-\right)\right)=x_{1}+\int_{t_{0}}^{t_{1}^{\prime}} \frac{f d \tau}{\tau^{1-\rho}}+J_{1}\left(x\left(t_{1}^{\prime}-\right)\right)
$$

and

$$
\begin{aligned}
{ }_{t_{0}}^{K} \mathcal{I}_{t_{1}^{\prime}+}^{2-q, \rho} x\left(t_{1}^{\prime}-\right) & ={ }_{t_{0}}^{K} \mathcal{I}_{t_{1-}^{\prime}}^{2-q, \rho} x\left(t_{1}^{\prime}-\right)+\bar{J}_{1}\left(x\left(t_{1}^{\prime}-\right)\right) \\
& =x_{2}+x_{1} \frac{\left(t_{1}^{\prime}\right)^{\rho}-\left(t_{0}\right)^{\rho}}{\rho}+\int_{t_{0}}^{t_{1}^{\prime}} \frac{\left(t_{1}^{\prime}\right)^{\rho}-\tau^{\rho}}{\rho} \frac{f d \tau}{\tau^{1-\rho}}+\bar{J}_{1}\left(x\left(t_{1}^{\prime}-\right)\right) .
\end{aligned}
$$

Therefore, the approximate solution of (1.1) is given as $t \in\left(t_{1}^{\prime}, t_{2}^{\prime}\right]$ by

$$
\begin{aligned}
\hat{x}(t)= & \frac{{ }_{t_{0}}^{K} \mathcal{I}_{t_{1}^{\prime}}^{2-q, \rho} x\left(t_{1}^{\prime}+\right)}{\Gamma(q-1)}\left[\frac{t^{\rho}-\left(t_{1}\right)^{\rho}}{\rho}\right]^{q-2}+\frac{{ }_{t_{0}}^{K} \mathcal{D}_{t_{1}^{\prime}}^{q-1, \rho} x\left(t_{1}^{\prime}+\right)}{\Gamma(q)}\left[\frac{t^{\rho}-\left(t_{1}^{\prime}\right)^{\rho}}{\rho}\right]^{q-1} \\
& +\frac{1}{\Gamma(q)} \int_{t_{1}^{\prime}}^{t}\left[\frac{t^{\rho}-\tau^{\rho}}{\rho}\right]^{q-1} \frac{f d \tau}{\tau^{1-\rho}} \text { for } t \in\left(t_{1}^{\prime}, t_{2}^{\prime}\right] \\
= & y\left(t_{1}^{\prime}, t\right)+\frac{\bar{J}_{1}\left(x\left(t_{1}^{\prime}-\right)\right)}{\Gamma(q-1)}\left[\frac{t^{\rho}-\left(t_{1}\right)^{\rho}}{\rho}\right]^{q-2}+\frac{J_{1}\left(x\left(t_{1}^{\prime}-\right)\right)}{\Gamma(q)}\left[\frac{t^{\rho}-\left(t_{1}^{\prime}\right)^{\rho}}{\rho}\right]^{q-1} \\
& \text { for } t \in\left(t_{1}^{\prime}, t_{2}^{\prime}\right],
\end{aligned}
$$


with the error $\hat{e}_{1}(t)=x(t)-\hat{x}(t)$ for $t \in\left(t_{1}^{\prime}, t_{2}^{\prime}\right]$, where $x(t)$ is the exact solution of (1.1). Moreover, by Lemmas 4.1 and 4.3 , the exact solution $x(t)$ of $(1.1)$ as $t \in\left(t_{1}^{\prime}, t_{2}^{\prime}\right]$ satisfies three conditions:

$$
\begin{aligned}
& \lim _{J_{1}\left(x\left(t_{1}^{\prime}-\right)\right) \rightarrow 0, \bar{J}_{1}\left(x\left(t_{1}^{\prime}-\right)\right) \rightarrow 0} x(t)=y\left(t_{0}, t\right) \quad \text { for } t \in\left(t_{1}^{\prime}, t_{2}^{\prime}\right], \\
& \lim _{\bar{J}_{1}\left(x\left(t_{1}^{\prime}-\right)\right) \rightarrow 0} x(t)=y\left(t_{0}, t\right)+\frac{J_{1}\left(x\left(t_{1}^{\prime}-\right)\right)}{\Gamma(q)}\left[\frac{t^{\rho}-\left(t_{1}^{\prime}\right)^{\rho}}{\rho}\right]^{q-1}+\xi J_{1}\left(x\left(t_{1}^{\prime}-\right)\right)\left[y\left(t_{1}^{\prime}, t\right)-y\left(t_{0}, t\right)\right] \\
& \text { for } t \in\left(t_{1}^{\prime}, t_{2}^{\prime}\right], \\
& \lim _{J_{1}\left(x\left(t_{1}^{\prime}-\right)\right) \rightarrow 0} x(t)=y\left(t_{0}, t\right)+\frac{\bar{J}_{1}\left(x\left(t_{1}^{\prime}-\right)\right)}{\Gamma(q-1)}\left[\frac{t^{\rho}-\left(t_{1}^{\prime}\right)^{\rho}}{\rho}\right]^{q-2}+\eta \bar{J}_{1}\left(x\left(t_{1}^{\prime}-\right)\right)\left[y\left(t_{1}^{\prime}, t\right)-y\left(t_{0}, t\right)\right] \\
& \text { for } t \in\left(t_{1}^{\prime}, t_{2}^{\prime}\right] .
\end{aligned}
$$

By (4.29)-(4.32), we get

$$
\begin{aligned}
\lim _{\substack{J_{1}\left(x\left(t_{1}^{\prime}-\right)\right) \rightarrow 0, \bar{J}_{1}\left(x\left(t_{1}^{\prime}-\right)\right) \rightarrow 0}} \hat{e}_{1}(t)=y\left(t_{0}, t\right)-y\left(t_{1}^{\prime}, t\right), \\
\lim _{\bar{J}_{1}\left(x\left(t_{1}^{\prime}-\right)\right) \rightarrow 0} \hat{e}_{1}(t)=\left[\xi J_{1}\left(x\left(t_{1}^{\prime}-\right)\right)-1\right]\left[y\left(t_{1}^{\prime}, t\right)-y\left(t_{0}, t\right)\right], \\
\lim _{J_{1}\left(x\left(t_{1}^{\prime}-\right)\right) \rightarrow 0} \hat{e}_{1}(t)=\left[\eta \bar{J}_{1}\left(x\left(t_{1}^{\prime}-\right)\right)-1\right]\left[y\left(t_{1}^{\prime}, t\right)-y\left(t_{0}, t\right)\right] .
\end{aligned}
$$

By (4.33)-(4.35), we obtain

$$
\hat{e}_{1}(t)=\left[\xi J_{1}\left(x\left(t_{1}^{\prime}-\right)\right)+\eta \bar{J}_{1}\left(x\left(t_{1}^{\prime}-\right)\right)-1\right]\left[y\left(t_{1}^{\prime}, t\right)-y\left(t_{0}, t\right)\right] \text { for } t \in\left(t_{1}^{\prime}, t_{2}^{\prime}\right] .
$$

By (4.29) and (4.36), we have

$$
\begin{aligned}
x(t)= & \hat{x}(t)+\hat{e}_{1}(t) \\
= & y\left(t_{0}, t\right)+\frac{J_{1}\left(x\left(t_{1}^{\prime}-\right)\right)}{\Gamma(q)}\left[\frac{t^{\rho}-\left(t_{1}^{\prime}\right)^{\rho}}{\rho}\right]^{q-1}+\frac{\bar{J}_{1}\left(x\left(t_{1}^{\prime}-\right)\right)}{\Gamma(q-1)}\left[\frac{t^{\rho}-\left(t_{1}\right)^{\rho}}{\rho}\right]^{q-2} \\
& +\left[\xi J_{1}\left(x\left(t_{1}^{\prime}-\right)\right)+\eta \bar{J}_{1}\left(x\left(t_{1}^{\prime}-\right)\right)\right]\left[y\left(t_{1}^{\prime}, t\right)-y\left(t_{0}, t\right)\right] \text { for } t \in\left(t_{1}^{\prime}, t_{2}^{\prime}\right] .
\end{aligned}
$$

Therefore the solution of (1.1) satisfies (4.25) as $t \in\left(t_{1}^{\prime}, t_{2}^{\prime}\right]$.

Next, for $t \in\left(t_{k}^{\prime}, t_{k+1}^{\prime}\right](k \in\{1,2, \ldots, M\})$, suppose that the solution of (1.1) satisfies

$$
\begin{aligned}
x(t)= & y\left(t_{0}, t\right)+\sum_{i=1}^{k_{1}} \frac{J_{i}\left(x\left(t_{i}-\right)\right)}{\Gamma(q)}\left[\frac{t^{\rho}-\left(t_{i}\right)^{\rho}}{\rho}\right]^{q-1}+\sum_{j=1}^{k_{2}} \frac{\bar{J}_{j}\left(x\left(\bar{t}_{j}-\right)\right)}{\Gamma(q-1)}\left[\frac{t^{\rho}-\left(\bar{t}_{j}\right)^{\rho}}{\rho}\right]^{q-2} \\
& +\xi \sum_{i=1}^{k_{1}} J_{i}\left(x\left(t_{i}-\right)\right)\left[y\left(t_{i}, t\right)-y\left(t_{0}, t\right)\right]+\eta \sum_{j=1}^{k_{2}} \bar{J}_{j}\left(x\left(\bar{t}_{j}-\right)\right)\left[y\left(\bar{t}_{j}, t\right)-y\left(t_{0}, t\right)\right]
\end{aligned}
$$

for $t \in\left(t_{k}^{\prime}, t_{k+1}^{\prime}\right]$. 
Using ${ }_{t_{0}}^{K} \mathcal{D}_{t}^{q-1, \rho}$ and ${ }_{t_{0}}^{K} \mathcal{I}_{t}^{2-q, \rho}$ to two sides of (4.38) respectively, we get

$$
\begin{aligned}
{ }_{t_{0}}^{K} \mathcal{D}_{t_{k+1}^{\prime}}^{q-1, \rho} x\left(t_{k+1}^{\prime}+\right) & ={ }_{t_{0}}^{K} \mathcal{D}_{t_{k+1}^{\prime}}^{q-1, \rho} x\left(t_{k+1}^{\prime}-\right)+\sum_{i=k_{1}+1}^{(k+1)_{1}} J_{i}\left(x\left(t_{i}-\right)\right) \\
& =x_{1}+\int_{t_{0}}^{t_{k+1}^{\prime}} \frac{f d \tau}{\tau^{1-\rho}}+\sum_{i=1}^{(k+1)_{1}} J_{i}\left(x\left(t_{i}-\right)\right)
\end{aligned}
$$

and

$$
\begin{aligned}
{ }_{t_{0}}^{K} \mathcal{I}_{t_{k+1}^{\prime}{ }^{\prime}}^{2-q, \rho} x\left(t_{k+1}^{\prime}+\right)= & { }_{t_{0}}^{K} \mathcal{I}_{t_{k+1}^{\prime}}^{2-q, \rho} x\left(t_{k+1}^{\prime}-\right)+\sum_{j=k_{2}+1}^{(k+1)_{2}} \bar{J}_{j}\left(x\left(\bar{t}_{j}-\right)\right) \\
= & x_{2}+x_{1} \frac{\left(t_{k+1}^{\prime}\right)^{\rho}-\left(t_{0}\right)^{\rho}}{\rho}+\int_{t_{0}}^{t_{k+1}^{\prime}} \frac{\left(t_{k+1}^{\prime}\right)^{\rho}-\tau^{\rho} f d \tau}{\rho} \frac{f d}{\tau^{1-\rho}} \\
& +\sum_{j=1}^{(k+1)_{2}} \bar{J}_{j}\left(x\left(\bar{t}_{j}-\right)\right)+\sum_{i=1}^{k_{1}} J_{i}\left(x\left(t_{i}-\right)\right) \frac{\left(t_{k+1}^{\prime}\right)^{\rho}-\left(t_{i}\right)^{\rho}}{\rho} .
\end{aligned}
$$

Therefore, the approximate solution of (1.1) as $t \in\left(t_{k+1}^{\prime}, t_{k+2}^{\prime}\right]$ is given by

$$
\begin{aligned}
\tilde{x}(t)= & \frac{{ }_{t_{0}}^{K} \mathcal{I}_{t_{k+1}^{\prime}}^{2-q, \rho} x\left(t_{k+1}^{\prime}+\right)}{\Gamma(q-1)}\left[\frac{t^{\rho}-\left(t_{k+1}^{\prime}\right)^{\rho}}{\rho}\right]^{q-2}+\frac{{ }_{t_{0}}^{K} \mathcal{D}_{t_{k+1}^{\prime}}^{q-1, \rho} x\left(t_{k+1}^{\prime}\right)}{\Gamma(q)}\left[\frac{t^{\rho}-\left(t_{k+1}^{\prime}\right)^{\rho}}{\rho}\right]^{q-1} \\
& +\frac{1}{\Gamma(q)} \int_{t_{k+1}^{\prime}}^{t}\left[\frac{t^{\rho}-\tau^{\rho}}{\rho}\right]^{q-1} \frac{f d \tau}{\tau^{1-\rho}} \text { for } t \in\left(t_{k+1}^{\prime}, t_{k+2}^{\prime}\right] \\
= & y\left(t_{k+1}^{\prime}, t\right)+\frac{\sum_{j=1}^{(k+1)_{2}} \bar{J}_{j}\left(x\left(\bar{t}_{j}-\right)\right)+\sum_{i=1}^{k_{1}} J_{i}\left(x\left(t_{i}-\right)\right) \frac{\left(t_{k+1}^{\prime}\right)^{\rho}-\left(t_{i}\right)^{\rho}}{\rho}}{\Gamma(q-1)}\left[\frac{t^{\rho}-\left(t_{k+1}^{\prime}\right)^{\rho}}{\rho}\right]^{q-2} \\
& +\frac{\sum_{i=1}^{(k+1)_{1}} J_{i}\left(x\left(t_{i}-\right)\right)}{\Gamma(q)}\left[\frac{t^{\rho}-\left(t_{k+1}^{\prime}\right)^{\rho}}{\rho}\right]^{q-1} \text { for } t \in\left(t_{k+1}^{\prime}, t_{k+2}^{\prime}\right],
\end{aligned}
$$

with $\hat{e}_{k+1}(t)=x(t)-\hat{x}(t)$ for $t \in\left(t_{k+1}^{\prime}, t_{k+2}^{\prime}\right]$, where $x(t)$ is the exact solution of (1.1). By (4.38), the exact solution of (1.1) satisfies

$$
\begin{aligned}
& \lim _{\substack{J_{i}\left(x\left(t_{i}-\right)\right) \rightarrow 0, \bar{j}_{j}\left(x\left(\bar{t}_{j}-\right)\right) \rightarrow 0 \\
\text { for all } i \text { and } j}} x(t)=y\left(t_{0}, t\right) \quad \text { for } t \in\left(t_{k+1}^{\prime}, t_{k+2}^{\prime}\right], \\
& \lim _{\substack{J_{i}\left(x\left(t_{i}-\right)\right) \rightarrow 0 \text { for all } i \in\left\{l_{1}+1, l_{1}+2, \ldots,(l+1)_{1}\right\}, \bar{J}_{j}\left(x\left(\bar{t}_{j}-\right)\right) \rightarrow 0 \text { for all } j \in\left\{l_{2}+1, l_{2}+2, \ldots,(l+1)_{2}\right\}}} x(t) \\
& =y\left(t_{0}, t\right)+\sum_{\substack{1 \leq i \leq(k+1)_{1} \text { and } \\
i \notin\left\{l_{1}+1, l_{1}+2, \ldots,(l+1)_{1}\right\}}} \frac{J_{i}\left(x\left(t_{i}-\right)\right)}{\Gamma(q)}\left[\frac{t^{\rho}-\left(t_{i}\right)^{\rho}}{\rho}\right]^{q-1} \\
& +y\left(t_{0}, t\right)+\sum_{\substack{1 \leq j \leq(k+1)_{2} \text { and } \\
j \notin\left\{l_{2}+1, l_{2}+2, \ldots,(l+1)_{2}\right\}}} \frac{\bar{J}_{j}\left(x\left(\bar{t}_{j}-\right)\right)}{\Gamma(q-1)}\left[\frac{t^{\rho}-\left(\bar{t}_{j}\right)^{\rho}}{\rho}\right]^{q-2} \\
& +\xi \sum_{\substack{1 \leq i \leq(k+1)_{1} \text { and } \\
i \notin\left\{l_{1}+1, l_{1}+2, \ldots,(l+1)_{1}\right\}}} J_{i}\left(x\left(t_{i}-\right)\right)\left[y\left(t_{i}, t\right)-y\left(t_{0}, t\right)\right]
\end{aligned}
$$




$$
+\eta \sum_{\substack{1 \leq j \leq(k+1)_{2} \text { and } \\ j \notin\left\{l_{2}+1, l_{2}+2, \ldots,(l+1)_{2}\right\}}} \bar{J}_{j}\left(x\left(\bar{t}_{j}-\right)\right)\left[y\left(\bar{t}_{j}, t\right)-y\left(t_{0}, t\right)\right]
$$

for $t \in\left(t_{k+1}^{\prime}, t_{k+2}^{\prime}\right], l=1,2, \ldots, k+1$.

By (4.41)-(4.43), we obtain

$$
\begin{aligned}
& \lim _{\substack{J_{i}\left(x\left(t_{i}-\right)\right) \rightarrow 0, \bar{J}_{j}\left(x\left(\bar{t}_{j}\right)\right) \rightarrow 0 \\
\text { for all } i \text { and } j}} \hat{e}_{k+1}(t)=-\left[y\left(t_{k+1}^{\prime}, t\right)-y\left(t_{0}, t\right)\right], \\
& \lim _{\substack{J_{i}\left(x\left(t_{i}-\right)\right) \rightarrow 0 \text { for all } i \in\left\{l_{1}+1, l_{1}+2, \ldots,(l+1)_{1}\right\}, \bar{J}_{j}\left(x\left(\bar{t}_{j}-\right)\right) \rightarrow 0 \text { for all } j \in\left\{l_{2}+1, l_{2}+2, \ldots,(l+1)_{2}\right\}}} \hat{e}_{k+1}(t) \\
& =-\left[y\left(t_{k+1}^{\prime}, t\right)-y\left(t_{0}, t\right)\right]+\xi \sum_{\substack{1 \leq i \leq(k+1)_{1} \text { and } \\
i \notin\left\{l_{1}+1, \ldots,(l+1)_{1}\right\}}} J_{i}\left(x\left(t_{i}-\right)\right)\left[y\left(t_{i}, t\right)-y\left(t_{0}, t\right)\right] \\
& +\eta \sum_{\substack{1 \leq j \leq(k+1)_{2} \text { and } \\
j \notin\left\{l l_{2}+1, \ldots,(l+1)_{2}\right\}}} \bar{J}_{j}\left(x\left(\bar{t}_{j}-\right)\right)\left[y\left(\bar{t}_{j}, t\right)-y\left(t_{0}, t\right)\right] \\
& +\sum_{\substack{1 \leq i \leq(k+1)_{1} \text { and } \\
i \notin\left\{l_{1}+1, l_{1}+2, \ldots,(l+1)_{1}\right\}}} \frac{J_{i}\left(x\left(t_{i}-\right)\right)}{\Gamma(q)}\left\{\left[\frac{t^{\rho}-\left(t_{i}\right)^{\rho}}{\rho}\right]^{q-1}-\left[\frac{t^{\rho}-\left(t_{k+1}^{\prime}\right)^{\rho}}{\rho}\right]^{q-1}\right\} \\
& +\sum_{\substack{1 \leq j \leq(k+1)_{2} \text { and } \\
j \notin\left\{l_{2}+1, l_{2}+2, \ldots,(l+1)_{2}\right\}}} \frac{\bar{J}_{j}\left(x\left(\bar{t}_{j}-\right)\right)}{\Gamma(q-1)}\left\{\left[\frac{t^{\rho}-\left(\bar{t}_{j}\right)^{\rho}}{\rho}\right]^{q-2}-\left[\frac{t^{\rho}-\left(t_{k+1}^{\prime}\right)^{\rho}}{\rho}\right]^{q-2}\right\} \\
& \quad-\sum_{\substack{1 \leq i \leq k_{1} \text { and } \\
i \notin\left\{l_{1}+1, l_{1}+2, \ldots,(l+1)_{1}\right\}}} \frac{J_{i}\left(x\left(t_{i}-\right)\right)}{\Gamma(q-1)} \frac{\left(t_{k+1}^{\prime}\right)^{\rho}-\left(t_{i}\right)^{\rho}}{\rho}\left[\frac{t^{\rho}-\left(t_{k+1}^{\prime}\right)^{\rho}}{\rho}\right]^{q-2} \\
& \text { for } t \in\left(t_{k+1}^{\prime}, t_{k+2}^{\prime}\right], l=1,2, \ldots, k+1 .
\end{aligned}
$$

By (4.44) and (4.45), we have

$$
\begin{aligned}
\hat{e}_{k+1}(t)= & -\left[y\left(t_{k+1}^{\prime}, t\right)-y\left(t_{0}, t\right)\right]-\sum_{i=1}^{k_{1}} \frac{J_{i}\left(x\left(t_{i}-\right)\right)}{\Gamma(q-1)} \frac{\left(t_{k+1}^{\prime}\right)^{\rho}-\left(t_{i}\right)^{\rho}}{\rho}\left[\frac{t^{\rho}-\left(t_{k+1}^{\prime}\right)^{\rho}}{\rho}\right]^{q-2} \\
& +\xi \sum_{i=1}^{(k+1)_{1}} J_{i}\left(x\left(t_{i}-\right)\right)\left[y\left(t_{i}, t\right)-y\left(t_{0}, t\right)\right] \\
& +\eta \sum_{j=1}^{(k+1)_{2}} \bar{J}_{j}\left(x\left(\bar{t}_{j}-\right)\right)\left[y\left(\bar{t}_{j}, t\right)-y\left(t_{0}, t\right)\right] \\
& +\sum_{i=1}^{(k+1)_{1}} \frac{J_{i}\left(x\left(t_{i}-\right)\right)}{\Gamma(q)}\left\{\left[\frac{t^{\rho}-\left(t_{i}\right)^{\rho}}{\rho}\right]^{q-1}-\left[\frac{t^{\rho}-\left(t_{k+1}^{\prime}\right)^{\rho}}{\rho}\right]^{q-1}\right\} \\
& +\sum_{j=1}^{(k+1)_{2}} \frac{\bar{J}_{j}\left(x\left(\bar{t}_{j}-\right)\right)}{\Gamma(q-1)}\left\{\left[\frac{t^{\rho}-\left(\bar{t}_{j}\right)^{\rho}}{\rho}\right]^{q-2}-\left[\frac{t^{\rho}-\left(t_{k+1}^{\prime}\right)^{\rho}}{\rho}\right]^{q-2}\right\} .
\end{aligned}
$$


By (4.41) and (4.46), we get

$$
\begin{aligned}
& x(t)= \hat{x}(t)+\hat{e}_{k+1}(t) \\
&= y\left(t_{0}, t\right)+\sum_{i=1}^{(k+1)_{1}} \frac{J_{i}\left(x\left(t_{i}-\right)\right)}{\Gamma(q)}\left[\frac{t^{\rho}-\left(t_{i}\right)^{\rho}}{\rho}\right]^{q-1}+\sum_{j=1}^{(k+1)_{2}} \frac{\bar{J}_{j}\left(x\left(\bar{t}_{j}-\right)\right)}{\Gamma(q-1)}\left[\frac{t^{\rho}-\left(\bar{t}_{j}\right)^{\rho}}{\rho}\right]^{q-2} \\
&+\xi \sum_{i=1}^{(k+1)_{1}} J_{i}\left(x\left(t_{i}-\right)\right)\left[y\left(t_{i}, t\right)-y\left(t_{0}, t\right)\right]+\eta \sum_{j=1}^{(k+1)_{2}} \bar{J}_{j}\left(x\left(\bar{t}_{j}-\right)\right)\left[y\left(\bar{t}_{j}, t\right)-y\left(t_{0}, t\right)\right] \\
& \text { for } t \in\left(t_{k+1}^{\prime}, t_{k+2}^{\prime}\right] .
\end{aligned}
$$

Thus the solution of (1.1) satisfies (4.25) as $t \in\left(t_{k+1}^{\prime}, t_{k+2}^{\prime}\right]$, and the necessity is proved.

Now we verify the sufficiency that (4.25) satisfies all the conditions of system (1.1). It is easy to find that (4.25) satisfies conditions (i)-(iv) by Lemmas 4.1 and 4.3, and it is similar with the proof of Lemma 4.1 to verify that (4.25) satisfies the condition of generalized fractional derivative, impulsive conditions, and initial conditions in (1.1). The proof is completed.

Corollary 4.5 Let $q \in(1,2]$ and $t_{0}, \rho>0$, and let $f:\left[t_{0}, T\right] \times \mathbb{R} \rightarrow \mathbb{R}$ be a function such that $f(\cdot, x(\cdot)) \in \hat{C}_{2-q, \rho}\left[t_{0}, T\right]$ for any $x(\cdot) \in \hat{C}_{2-q, \rho}\left[t_{0}, T\right]$.

If $x(\cdot) \in I C\left(\left[t_{0}, T\right], \mathbb{R}\right)$, then $x(t)$ is a solution of $(1.4)$ if, and only if, $x(t)$ satisfies the following integral equation:

$$
x(t)=\left\{\begin{array}{l}
y\left(t_{0}, t\right) \quad \text { for } t \in\left(t_{0}, t_{1}\right], \\
y\left(t_{0}, t\right)+\sum_{i=1}^{k} \frac{J_{i}\left(x\left(t_{i}-\right)\right)}{\Gamma(q)}\left[\frac{t^{\rho}-\left(t_{i}\right)^{\rho}}{\rho}\right]^{q-1}+\sum_{i=1}^{k} \frac{\bar{J}_{i}\left(x\left(t_{i}-\right)\right)}{\Gamma(q-1)}\left[\frac{t^{\rho}-\left(t_{i}\right)^{\rho}}{\rho}\right]^{q-2} \\
\quad+\sum_{i=1}^{k}\left[\xi J_{i}\left(x\left(t_{i}-\right)\right)+\eta \bar{J}_{i}\left(x\left(t_{i}-\right)\right)\right]\left[y\left(t_{i}, t\right)-y\left(t_{0}, t\right)\right] \\
\quad \text { for } t \in\left(t_{k}, t_{k+1}\right], k=1,2, \ldots, m,
\end{array}\right.
$$

where $\xi$ and $\eta$ are two arbitrary constants.

\section{Examples}

In this section, we consider the following IVP of three IFrDEs:

$$
\begin{aligned}
& \left\{\begin{array}{l}
{ }_{1}^{K} \mathcal{D}_{t}^{\frac{3}{2}, \rho} x(t)=x(t), \quad t \in(1,5], t \neq 3 \\
{ }_{1} \mathcal{D}_{3^{+}}^{\frac{1}{2}, \rho} x\left(3^{+}\right)-{ }_{1}^{K} \mathcal{D}_{3^{-}}^{\frac{1}{2}, \rho} x\left(3^{-}\right)=1, \\
\left.{ }_{1}^{K} \mathcal{D}_{t}^{\frac{1}{2}, \rho} x(t)\right|_{t \rightarrow 1+}=1,\left.\quad{ }_{1}^{K} \mathcal{I}_{t}^{\frac{1}{2}, \rho} x(t)\right|_{t \rightarrow 1+}=0,
\end{array}\right. \\
& \left\{\begin{array}{l}
{ }_{1}^{K} \mathcal{D}_{t}^{\frac{3}{2}, \rho} x(t)=x(t), \quad t \in(1,5], t \neq 3 \\
{ }_{1} \mathcal{I}_{3^{+}}^{\frac{1}{2}, \rho} x\left(3^{+}\right)-{ }_{1}^{K} \mathcal{I}_{3^{-}}^{\frac{1}{2}, \rho} x\left(3^{-}\right)=1, \\
\left.{ }_{1}^{K} \mathcal{D}_{t}^{\frac{1}{2}, \rho} x(t)\right|_{t \rightarrow 1+}=1,\left.\quad{ }_{1}^{K} \mathcal{I}_{t}^{\frac{1}{2}, \rho} x(t)\right|_{t \rightarrow 1+}=0,
\end{array}\right. \\
& \left\{\begin{array}{l}
{ }_{1}^{K} \mathcal{D}_{t}^{\frac{3}{2}, \rho} x(t)=x(t), \quad t \in(1,5], t \neq 3 \\
{ }_{1}^{K} \mathcal{D}_{3^{+}}^{\frac{1}{2}, \rho} x\left(3^{+}\right)-{ }_{1}^{K} \mathcal{D}_{3^{-}}^{\frac{1}{2}, \rho} x\left(3^{-}\right)=1, \\
{ }_{1}^{K} \mathcal{I}_{3^{+}}^{\frac{1}{2}, \rho} x\left(3^{+}\right)-{ }_{1}^{K} \mathcal{I}_{3^{-}}^{\frac{1}{2}, \rho} x\left(3^{-}\right)=1, \\
\left.{ }_{1}^{K} \mathcal{D}_{t}^{\frac{1}{2}, \rho} x(t)\right|_{t \rightarrow 1+}=1,\left.\quad{ }_{1}^{K} \mathcal{I}_{t}^{\frac{1}{2}, \rho} x(t)\right|_{t \rightarrow 1+}=0 .
\end{array}\right.
\end{aligned}
$$


By Lemma 4.1, Lemma 4.3, and Corollary 4.5, the equivalent integral equations of three systems $(5.1)-(5.3)$ as $t \in(1,3]$ are identical as follows:

$$
x(t)=\frac{2}{\sqrt{\pi}}\left[\frac{t^{\rho}-1}{\rho}\right]^{\frac{1}{2}}+\frac{2}{\sqrt{\pi}} \int_{1}^{t}\left[\frac{t^{\rho}-\tau^{\rho}}{\rho}\right]^{\frac{1}{2}} \frac{x(\tau) d \tau}{\tau^{1-\rho}} \quad \text { for } t \in(1,3]
$$

and the equivalent integral equations of three systems (5.1)-(5.3) as $t \in(3,5]$ are respectively given by

$$
\begin{aligned}
x(t)= & \frac{2}{\sqrt{\pi}}\left[\frac{t^{\rho}-1}{\rho}\right]^{\frac{1}{2}}+\frac{2}{\sqrt{\pi}} \int_{1}^{t}\left[\frac{t^{\rho}-\tau^{\rho}}{\rho}\right]^{\frac{1}{2}} \frac{x(\tau) d \tau}{\tau^{1-\rho}}+\frac{2}{\sqrt{\pi}}\left[\frac{t^{\rho}-3^{\rho}}{\rho}\right]^{\frac{1}{2}} \\
& +\xi\left\{\frac{\frac{3^{\rho}-1}{\rho}+\int_{1}^{3} \frac{3^{\rho}-\tau^{\rho}}{\rho} \frac{x(\tau) d \tau}{\tau^{1-\rho}}}{\sqrt{\pi}}\left[\frac{t^{\rho}-3^{\rho}}{\rho}\right]^{-\frac{1}{2}}+\frac{1+\int_{1}^{3} \frac{x(\tau) d \tau}{\tau^{1-\rho}}}{\sqrt{\pi} / 2}\left[\frac{t^{\rho}-3^{\rho}}{\rho}\right]^{\frac{1}{2}}\right. \\
& +\frac{2}{\sqrt{\pi}} \int_{3}^{t}\left[\frac{t^{\rho}-\tau^{\rho}}{\rho}\right]^{\frac{1}{2}} \frac{x(\tau) d \tau}{\tau^{1-\rho}}-\frac{2}{\sqrt{\pi}}\left[\frac{t^{\rho}-1}{\rho}\right]^{\frac{1}{2}} \\
& -\frac{2}{\sqrt{\pi}} \int_{1}^{t}\left[\frac{t^{\rho}-\tau^{\rho}}{\rho}\right]^{\frac{1}{2}} \frac{x(\tau) d \tau}{\tau^{1-\rho}}
\end{aligned}
$$

for $t \in(3,5]$,

$$
\begin{aligned}
x(t)= & \frac{2}{\sqrt{\pi}}\left[\frac{t^{\rho}-1}{\rho}\right]^{\frac{1}{2}}+\frac{2}{\sqrt{\pi}} \int_{1}^{t}\left[\frac{t^{\rho}-\tau^{\rho}}{\rho}\right]^{\frac{1}{2}} \frac{x(\tau) d \tau}{\tau^{1-\rho}}+\frac{1}{\sqrt{\pi}}\left[\frac{t^{\rho}-3^{\rho}}{\rho}\right]^{-\frac{1}{2}} \\
& +\eta\left\{\frac{\frac{3^{\rho}-1}{\rho}+\int_{1}^{3} \frac{3^{\rho}-\tau^{\rho}}{\rho} \frac{x(\tau) d \tau}{\tau^{1-\rho}}}{\sqrt{\pi}}\left[\frac{t^{\rho}-3^{\rho}}{\rho}\right]^{-\frac{1}{2}}+\frac{1+\int_{1}^{3} \frac{x(\tau) d \tau}{\tau^{1-\rho}}}{\sqrt{\pi} / 2}\left[\frac{t^{\rho}-3^{\rho}}{\rho}\right]^{\frac{1}{2}}\right. \\
& +\frac{2}{\sqrt{\pi}} \int_{3}^{t}\left[\frac{t^{\rho}-\tau^{\rho}}{\rho}\right]^{\frac{1}{2}} \frac{x(\tau) d \tau}{\tau^{1-\rho}}-\frac{2}{\sqrt{\pi}}\left[\frac{t^{\rho}-1}{\rho}\right]^{\frac{1}{2}} \\
& \left.-\frac{2}{\sqrt{\pi}} \int_{1}^{t}\left[\frac{t^{\rho}-\tau^{\rho}}{\rho}\right]^{\frac{1}{2}} \frac{x(\tau) d \tau}{\tau^{1-\rho}}\right\}
\end{aligned}
$$

for $t \in(3,5]$,

$$
\begin{aligned}
x(t)= & \frac{2}{\sqrt{\pi}}\left[\frac{t^{\rho}-1}{\rho}\right]^{\frac{1}{2}}+\frac{2}{\sqrt{\pi}} \int_{1}^{t}\left[\frac{t^{\rho}-\tau^{\rho}}{\rho}\right]^{\frac{1}{2}} \frac{x(\tau) d \tau}{\tau^{1-\rho}} \\
& +\frac{2}{\sqrt{\pi}}\left[\frac{t^{\rho}-3^{\rho}}{\rho}\right]^{\frac{1}{2}}+\frac{1}{\sqrt{\pi}}\left[\frac{t^{\rho}-3^{\rho}}{\rho}\right]^{-\frac{1}{2}} \\
& +[\xi+\eta]\left\{\frac{\frac{3^{\rho}-1}{\rho}+\int_{1}^{3} \frac{3^{\rho}-\tau^{\rho}}{\rho} \frac{x(\tau) d \tau}{\tau^{1-\rho}}}{\sqrt{\pi}}\left[\frac{t^{\rho}-3^{\rho}}{\rho}\right]^{-\frac{1}{2}}+\frac{1+\int_{1}^{3} \frac{x(\tau) d \tau}{\tau^{1-\rho}}}{\sqrt{\pi} / 2}\left[\frac{t^{\rho}-3^{\rho}}{\rho}\right]^{\frac{1}{2}}\right. \\
& +\frac{2}{\sqrt{\pi}} \int_{3}^{t}\left[\frac{t^{\rho}-\tau^{\rho}}{\rho}\right]^{\frac{1}{2}} \frac{x(\tau) d \tau}{\tau^{1-\rho}}-\frac{2}{\sqrt{\pi}}\left[\frac{t^{\rho}-1}{\rho}\right]^{\frac{1}{2}} \\
& \left.-\frac{2}{\sqrt{\pi}} \int_{1}^{t}\left[\frac{t^{\rho}-\tau^{\rho}}{\rho}\right]^{\frac{1}{2}} \frac{x(\tau) d \tau}{\tau^{1-\rho}}\right\}
\end{aligned}
$$

for $t \in(3,5]$,

where $\xi$ and $\eta$ in (5.5)-(5.7) are two arbitrary constants. 
Next we realize numerical simulation of (5.4) and (5.5)-(5.7) by using the Euler method with variable step size to give some solution trajectories of three systems (5.1)-(5.3) with given $\rho$, respectively.

Figures 1-4 denote the solution trajectories of (5.1) with $\rho=0.1,0.5,1,2$, respectively. Moreover, in these figures three curves ' $x i=0,1,-1$ ', which are drawn by numerical simulation of (5.4) - (5.5) with $\xi=0,1,-1$, respectively, represent three solutions of (5.1) with the corresponding $\rho$.

Figures 5-8 denote the solution trajectories of (5.2) with $\rho=0.1,0.5,1,2$, respectively. Moreover, in these figures three curves 'eta $=0,1,-1$ ', which are drawn by numerical simulation of (5.4) and (5.6) with $\eta=0,1,-1$, respectively, represent three solutions of (5.2) with the corresponding $\rho$.

Figures 9-12 denote the solution trajectories of (5.3) with $\rho=0.1,0.5,1,2$, respectively. Moreover, in these figures five curves ' $x i+$ eta $=2,1,0,-1,-2$ ', which are drawn by nu-

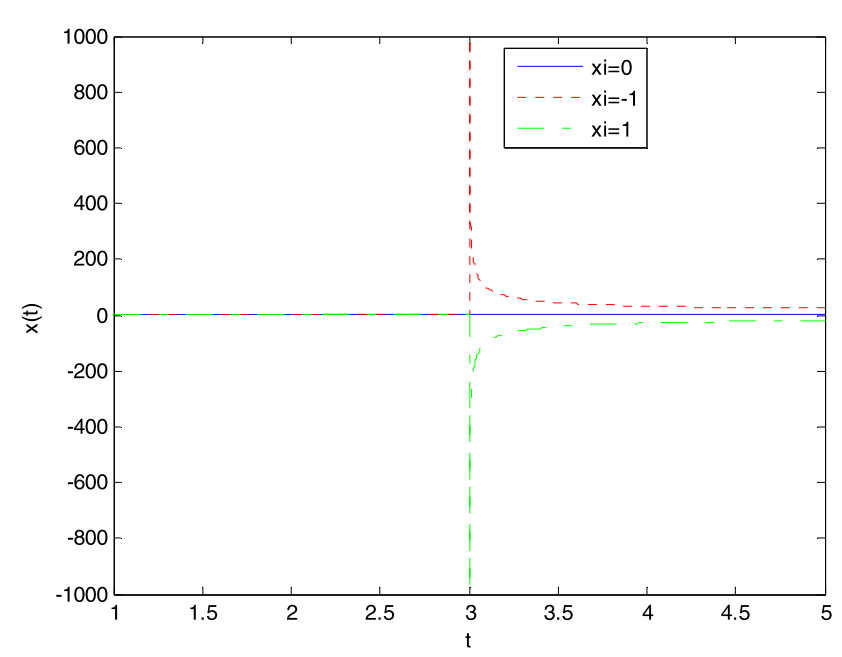

Figure 1 The solution trajectory of system (5.1) with $\rho=0.1$

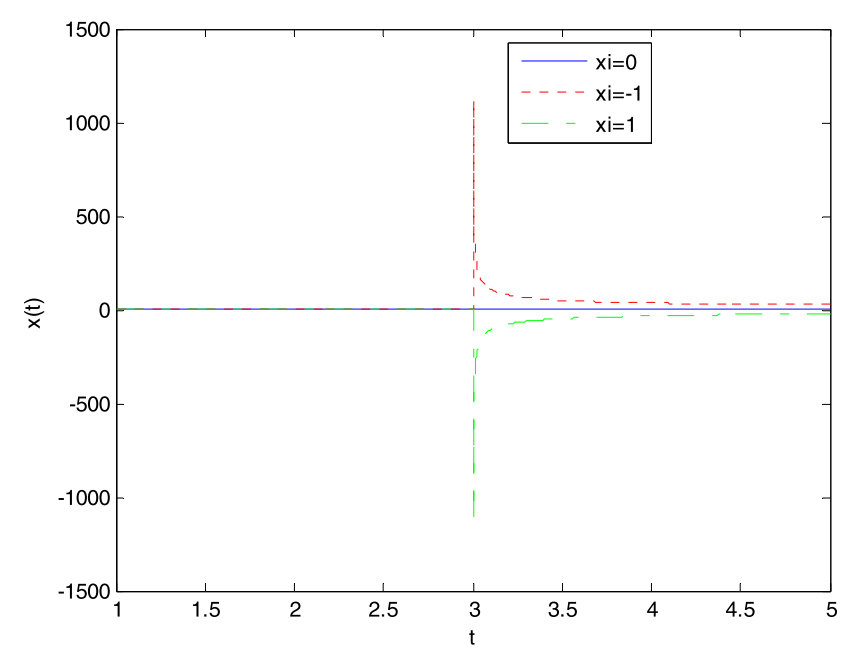

Figure 2 The solution trajectory of system (5.1) with $\rho=0.5$ 


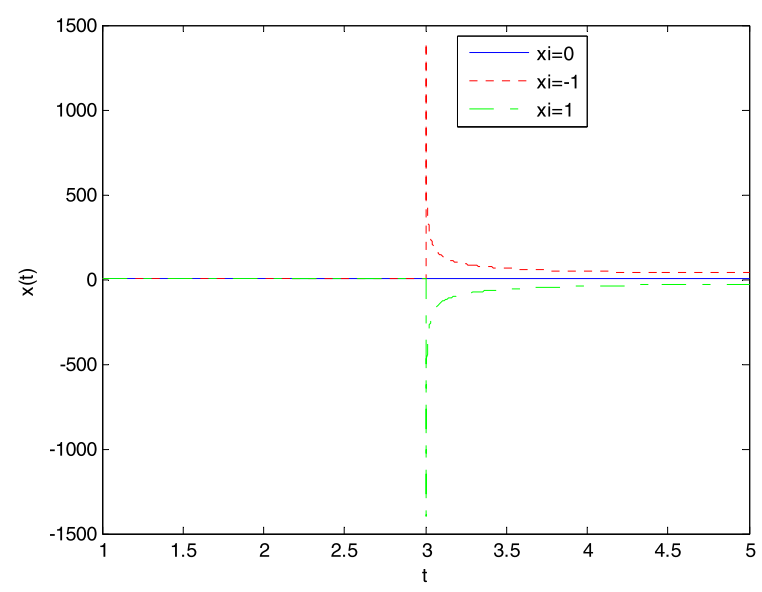

Figure 3 The solution trajectory of system (5.1) with $\rho=1$

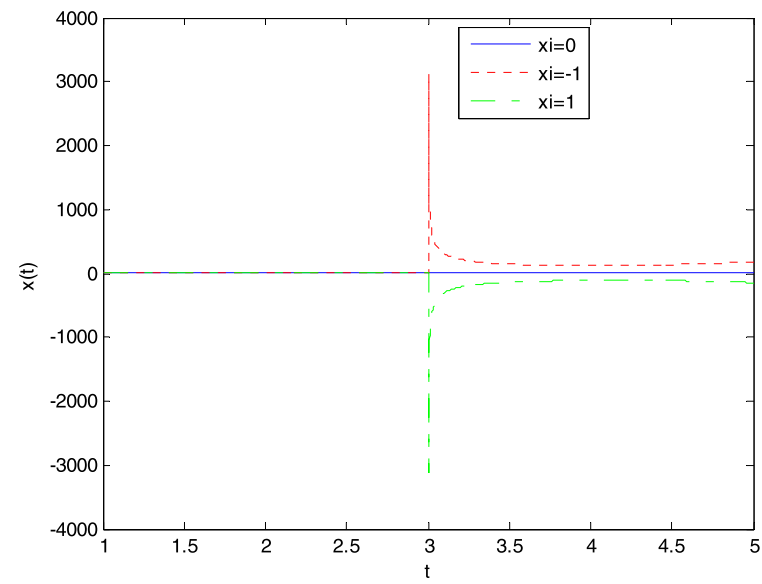

Figure 4 The solution trajectory of system (5.1) with $\rho=2$

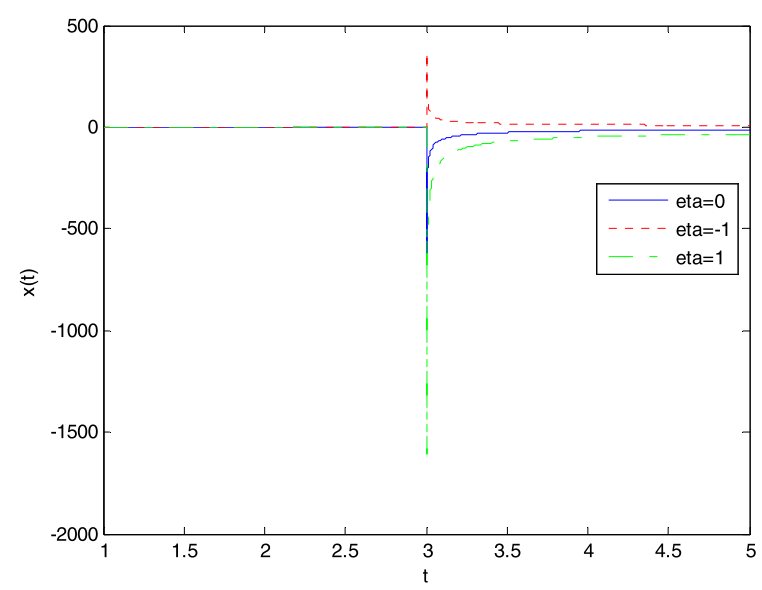

Figure 5 The solution trajectory of system (5.2) with $\rho=0.1$ 


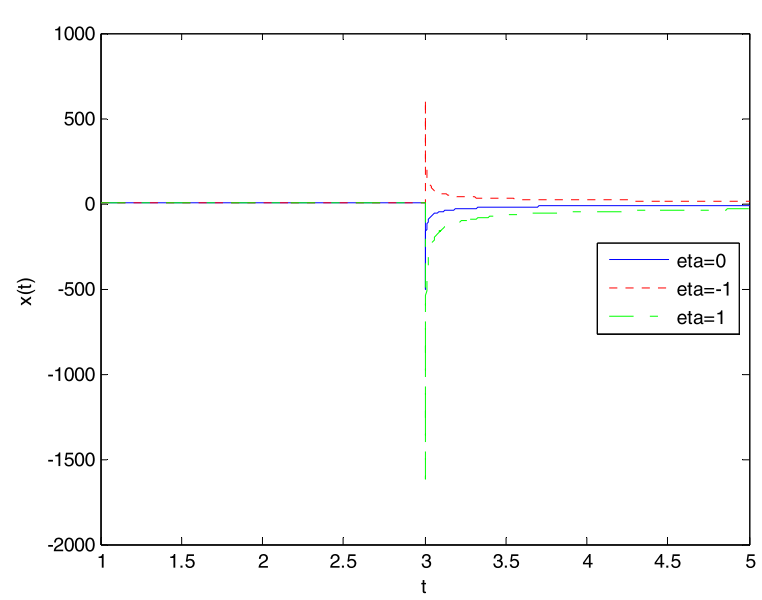

Figure 6 The solution trajectory of system (5.2) with $\rho=0.5$

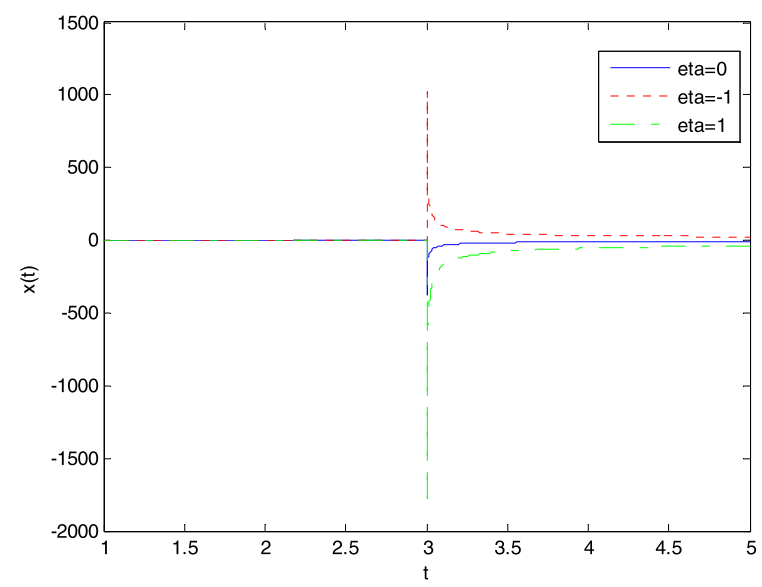

Figure 7 The solution trajectory of system (5.2) with $\rho=1$

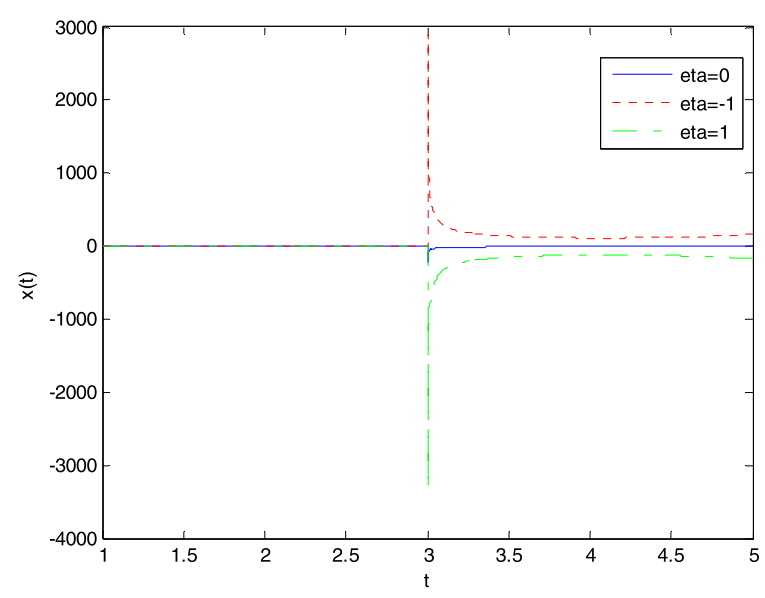

Figure 8 The solution trajectory of system (5.2) with $\rho=2$ 


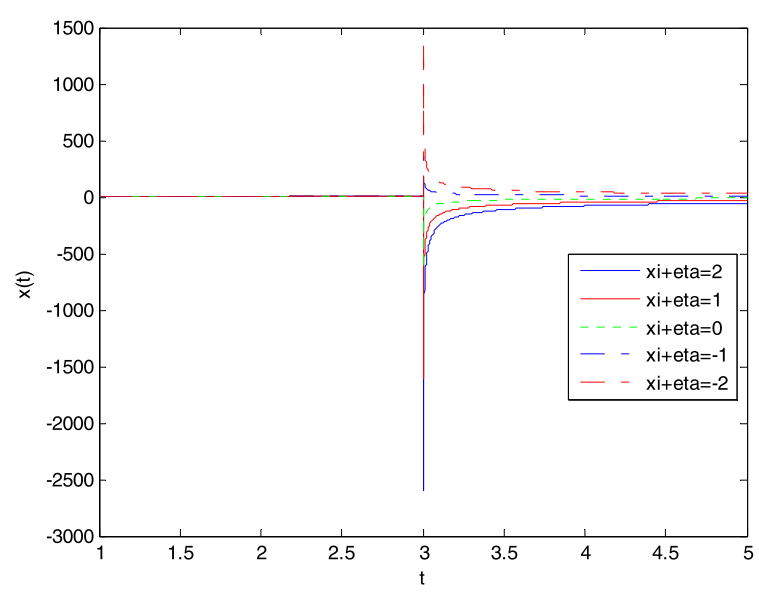

Figure 9 The solution trajectory of system (5.3) with $\rho=0.1$

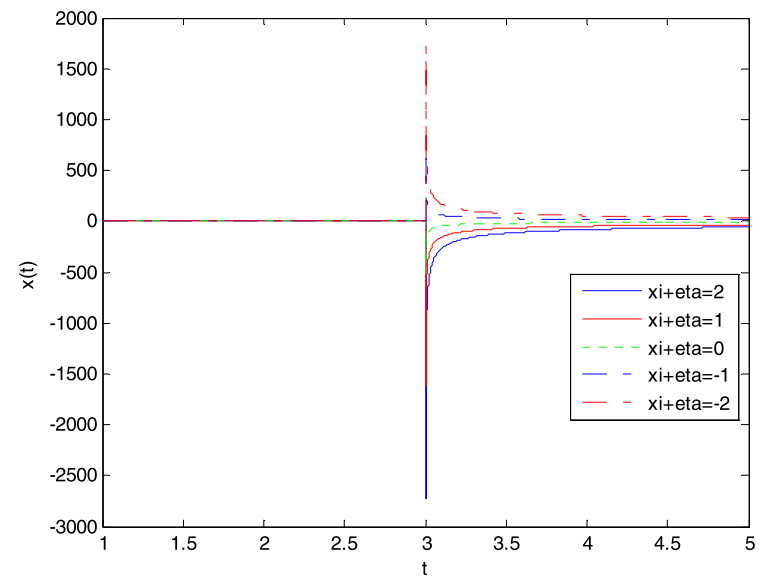

Figure 10 The solution trajectory of system (5.3) with $\rho=0.5$

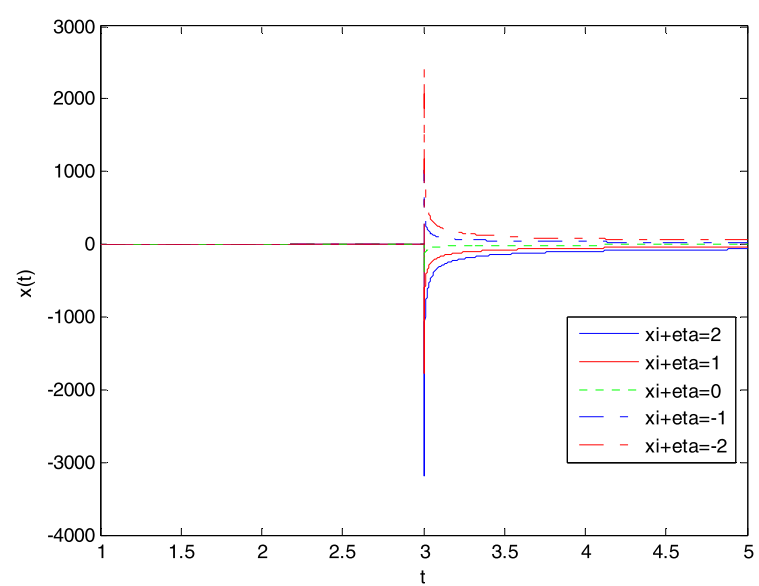

Figure 11 The solution trajectory of system (5.3) with $\rho=1$ 


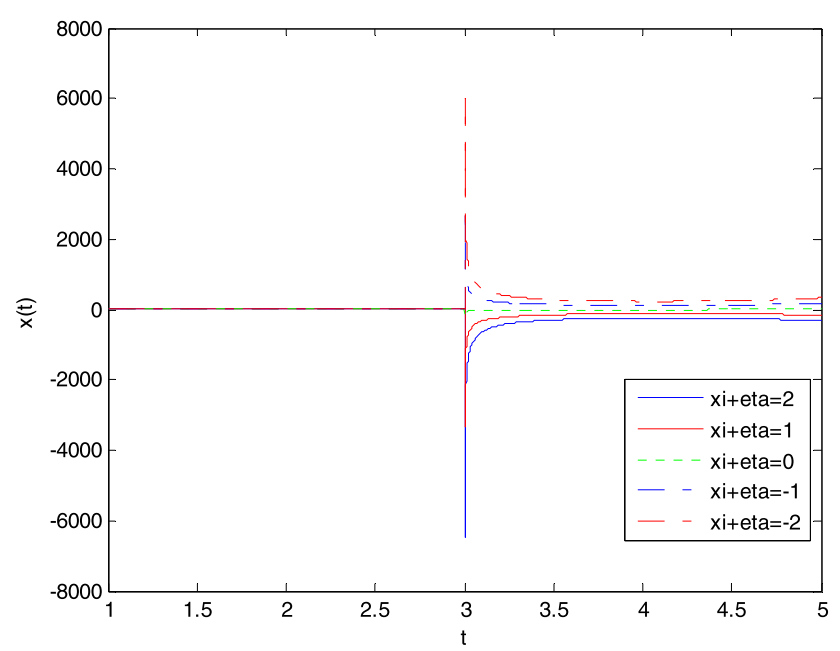

Figure 12 The solution trajectory of system (5.3) with $\rho=2$

merical simulation of (5.4) and (5.7) with $\xi+\eta=2,1,0,-1,-2$, respectively, represent five solutions of (5.3) with the corresponding $\rho$.

\section{Conclusion}

The systems of impulsive high order fractional differential equations can involve one or two kinds of impulses. As a result, their equivalent integral equations include one or two arbitrary constants which uncover the non-uniqueness of solution for the systems of impulsive high order fractional differential equations.

\section{Acknowledgements}

The author is deeply grateful to the anonymous referees for their kind comments, correcting errors, and improving written language, which have been very useful for improving the quality of this paper.

\section{Funding}

The work described in this paper is financially supported by the National Natural Science Foundation of China (Grant No. 21576033, 21636004)

Availability of data and materials

Not applicable.

\section{Competing interests}

The author declares that he has no competing interests.

\section{Authors' contributions}

The author wrote the first version of the manuscript and approved the final manuscript by himself.

\section{Publisher's Note}

Springer Nature remains neutral with regard to jurisdictional claims in published maps and institutional affiliations.

Received: 28 October 2019 Accepted: 3 February 2020 Published online: 22 February 2020

\section{References}

1. Deng, W: Smoothness and stability of the solutions for nonlinear fractional differential equations. Nonlinear Anal. TMA 72, 1768-1777 (2010)

2. Wu, G.C., Deng, Z.G., Baleanu, D., Zeng, D.Q.: New variable-order fractional chaotic systems for fast image encryption. Chaos 29, 083103 (2019)

3. Wu, G.C., Baleanu, D., Xie, H.P., Zeng, S.D.: Discrete fractional diffusion equation of chaotic order. Int. J. Bifurc. Chaos 26, 1650013 (2016)

4. Shiri, B., Baleanu, D.: System of fractional differential algebraic equations with applications. Chaos Solitons Fractals $120,203-212(2019)$ 
5. Alkahtani, B.S.T.: Chua's circuit model with Atangana-Baleanu derivative with fractional order. Chaos Solitons Fractals $89,547-551(2016)$

6. Koca, I.: Analysis of rubella disease model with non-local and non-singular fractional derivatives. Int. J. Optim. Control 8, 17-25 (2018)

7. Gómez-Aguilar, J.: Irving-Mullineux oscillator via fractional derivatives with Mittag-Leffler kernel. Chaos Solitons Fractals 95, 179-186 (2017)

8. Tateishi, A.A., Ribeiro, H.V., Lenzi, E.K.: The role of fractional time-derivative operators on anomalous diffusion. Front. Phys. 5, 1-9 (2017)

9. Morales-Delgado, V., Gómez-Aguilar, J., Taneco-Hernandez, M.: Analytical solutions for the motion of a charged particle in electric and magnetic fields via non-singular fractional derivatives. Eur. Phys. J. Plus 132, 527 (2017)

10. Dadkhah, E., Shiri, B., Ghaffarzadeh, H., Baleanu, D.: Visco-elastic dampers in structural buildings and numerica solution with spline collocation methods. J. Appl. Math. Comput. (2019). https://doi.org/10.1007/s12190-019-01307-5

11. Alijani, Z., Baleanu, D., Shiri, B., Wu, G.C.: Spline collocation methods for systems of fuzzy fractional differential equations. Chaos Solitons Fractals (2019). https://doi.org/10.1016/j.chaos.2019.109510

12. Gómez-Aguilar, J.F., Atangana, A.: Fractional derivatives with the power-law and the Mittag-Leffler kernel applied to the nonlinear Baggs-Freedman model. Fractal Fract. 2, 1-14 (2018)

13. Coronel-Escamilla, A., Gómez-Aguilar, J., Torres, L., Escobar-Jiménez, R.: A numerical solution for a variable-order reaction-diffusion model by using fractional derivatives with non-local and non-singular kernel. Phys. A, Stat. Mech. Appl. 491, 406-424 (2018)

14. Zuniga-Aguilar, C., Gómez-Aguilar, J., Escobar-Jiménez, R., Romero-Ugalde, H.: Robust control for fractional variable-order chaotic systems with non-singular kernel. Eur. Phys. J. Plus 133, 1-13 (2018)

15. Shiri, B., Baleanu, D.: Numerical solution of some fractional dynamical systems in medicine involving non-singular kernel with vector order. Results Nonlinear Anal. 2, 160-168 (2019)

16. Voyiadjis, G.Z., Sumelka, W.: Brain modelling in the framework of anisotropic hyperelasticity with time fractional damage evolution governed by the Caputo-Almeida fractional derivative. J. Mech. Behav. Biomed. Mater. 89, 209-216 (2019)

17. Wu, G.C., Baleanu, D., Zeng, S.D., Deng, Z.G.: Discrete fractional diffusion equation. Nonlinear Dyn. 80, 281-286 (2015)

18. Kilbas, A.A., Srivastava, H.H., Trujillo, J.J.: Theory and Applications of Fractional Differential Equations. Elsevier, Amsterdam (2006)

19. Baleanu, D., Diethelm, K., Scalas, E., Trujillo, J.J.: Fractional Calculus Models and Numerical Methods, Series on Complexity, Nonlinearity and Chaos. World Scientific, Singapore (2012)

20. Hilfer, R.: Applications of Fractional Calculus in Physics. World Scientific, Singapore (2000)

21. Hilfer, R., Luchko, Y., Tomovski, U.: Operational method for the solution of fractional differential equations with generalized Riemann-Liouville fractional derivatives. Fract. Calc. Appl. Anal. 12, 299-318 (2009)

22. Katugampola, U.N.: New approach to a generalized fractional integral. Appl. Math. Comput. 218, 860-865 (2011)

23. Katugampola, U.N.: A new approach to generalized fractional derivatives. Bull. Math. Anal. Appl. 6, 1-15 (2014)

24. Abdon, A., Dumitru, B.: New fractional derivatives with nonlocal and non-singular kernel; theory and application to heat transfer model. Therm. Sci. 20, 763-769 (2016)

25. Gou, H., Li, B.: Study on the mild solution of Sobolev type Hilfer fractional evolution equations with boundary conditions. Chaos Solitons Fractals 112, 168-179 (2018)

26. Zhang, X.: Non-uniqueness of solution for initial value problem of impulsive Caputo-Katugampola fractional differential differential equations. Int. J. Dyn. Syst. Differ. Equ. (in press). https://www.inderscience.com/info/ingeneral/ forthcoming.php?jcode=ijdsde

27. Harrat, A., Nieto, J.J., Debbouche, A.: Solvability and optimal controls of impulsive Hilfer fractional delay evolution inclusions with Clarke subdifferential. J. Comput. Appl. Math. 344, 725-737 (2018)

28. Debbouche, A., Antonov, V:: Approximate controllability of semilinear Hilfer fractional differential inclusions with impulsive control inclusion conditions in Banach spaces. Chaos Solitons Fractals 102, 140-148 (2017)

29. Jarad, F., Abdeljawad, T., Baleanu, D.: On the generalized fractional derivatives and their Caputo modification. J. Nonlinear Sci. Appl. 10, 2607-2619 (2017)

30. Zeng, S., Baleanu, D., Bai, Y., Wu, G.: Fractional differential equations of Caputo-Katugampola type and numerical solutions. Appl. Math. Comput. 315, 549-554 (2017)

31. Almeida, R., Malinowska, A.B., Odzijewicz, T.: Fractional differential equations with dependence on the Caputo-Katugampola derivative. J. Comput. Nonlinear Dyn. 11, 061017 (2016)

32. Baleanu, D., Shiri, B., Srivastava, H.M., Qurashi, Al.M.: A Chebyshev spectral method based on operational matrix for fractional differential equations involving non-singular Mittag-Leffler kernel. Adv. Differ. Equ. 2018, 353 (2018)

33. Baleanu, D., Shiri, B.: Collocation methods for fractional differential equations involving non-singular kernel. Chaos Solitons Fractals 116, 136-145 (2018)

34. Anderson, D.R., Ulness, D.J.: Properties of the Katugampola fractional derivative with potential application in quantum mechanics. J. Math. Phys. 56, 063502 (2015)

35. Thaiprayoon, C., Ntouyas, S.K., Tariboon, J.: On the nonlocal Katugampola fractional integral conditions for fractional Langevin equation. Adv. Differ. Equ. 2015, 374 (2015)

36. Lakshmikantham, V., Bainov, D.D., Simeonov, P.S.: Theory of Impulsive Differential Equations. World Scientific, Singapore (1989)

37. Agarwal, R., Hristova, S., O'Regan, D.: A survey of Lyapunov functions, stability and impulsive Caputo fractional differential equations. Fract. Calc. Appl. Anal. 19, 290-318 (2016)

38. Wang, J.R., Feckan, M., Zhou, Y.: A survey on impulsive fractional differential equations. Fract. Calc. Appl. Anal. 19, 806-831 (2016)

39. Zhang, X., Zhang, X., Zhang, M.: On the concept of general solution for impulsive differential equations of fractional order $q \in(0,1)$. Appl. Math. Comput. 247, 72-89 (2014)

40. Zhang, X.: On the concept of general solutions for impulsive differential equations of fractional order $q \in(1,2)$. Appl. Math. Comput. 268, 103-120 (2015)

41. Stamova, I., Stamov, G.: Stability analysis of impulsive functional systems of fractional order. Commun. Nonlinear Sci. Numer. Simul. 19(3), 702-709 (2014) 
42. Wang, G., Ahmad, B., Zhang, L., Nieto, J.J.: Comments on the concept of existence of solution for impulsive fractional differential equations. Commun. Nonlinear Sci. Numer. Simul. 19, 401-403 (2014)

43. Feckan, M., Zhou, Y., Wang, J.R.: Response to "Comments on the concept of existence of solution for impulsive fractional differential equations [Commun Nonlinear Sci Numer Simul 2014; 19:401-3.]". Commun. Nonlinear Sci. Numer. Simul. 19, 4213-4215 (2014)

44. Zhang, X., Shu, T., Cao, H., Liu, Z., Ding, W.: The general solution for impulsive differential equations with Hadamard fractional derivative of order $q \in(1,2)$. Adv. Differ. Equ. 2016, 14 (2016)

45. Fan, Z: A short note on the solvability of impulsive fractional differential equations with Caputo derivatives. Appl. Math. Lett. 38, 14-19 (2014)

46. Wu, G.C., Zeng, D.Q., Baleanu, D.: Fractional impulsive differential equations: exact solutions, integral equations and short memory case. Fract. Calc. Appl. Anal. 22, 180-192 (2019)

Submit your manuscript to a SpringerOpen ${ }^{\circ}$ journal and benefit from:

- Convenient online submission

Rigorous peer review

Open access: articles freely available online

- High visibility within the field

- Retaining the copyright to your article

Submit your next manuscript at $\gg$ springeropen.com 